\title{
Determinants of fruit and vegetable consumption among children and adolescents: a review of the literature. Part II: qualitative studies
}

\author{
Rikke Krølner ${ }^{1 *}$, Mette Rasmussen ${ }^{1}$, Johannes Brug $^{2}$, Knut-Inge Klepp $^{3}$, Marianne Wind $^{4}$ and Pernille Due
}

\begin{abstract}
Background: Large proportions of children do not fulfil the World Health Organization recommendation of eating at least 400 grams of fruit and vegetables (FV) per day. To promote an increased FV intake among children it is important to identify factors which influence their consumption. Both qualitative and quantitative studies are needed. Earlier reviews have analysed evidence from quantitative studies. The aim of this paper is to present a systematic review of qualitative studies of determinants of children's FV intake.

Methods: Relevant studies were identified by searching Anthropology Plus, Cinahl, CSA illumine, Embase, International Bibliography of the Social Sciences, Medline, PsycINFO, and Web of Science using combinations of synonyms for FV intake, children/adolescents and qualitative methods as search terms. The literature search was completed by December 1st 2010. Papers were included if they applied qualitative methods to investigate 6-18year-olds' perceptions of factors influencing their FV consumption. Quantitative studies, review studies, studies reported in other languages than English, and non-peer reviewed or unpublished manuscripts were excluded. The papers were reviewed systematically using standardised templates for summary of papers, quality assessment, and synthesis of findings across papers.

Results: The review included 31 studies, mostly based on US populations and focus group discussions. The synthesis identified the following potential determinants for FV intake which supplement the quantitative knowledge base: Time costs; lack of taste guarantee; satiety value; appropriate time/occasions/settings for eating $\mathrm{FV}$; sensory and physical aspects; variety, visibility, methods of preparation; access to unhealthy food; the symbolic value of food for image, gender identity and social interaction with peers; short term outcome expectancies.

Conclusions: The review highlights numerous potential determinants which have not been investigated thoroughly in quantitative studies. Future large scale quantitative studies should attempt to quantify the importance of these factors. Further, mechanisms behind gender, age and socioeconomic differences in FV consumption are proposed which should be tested quantitatively in order to better tailor interventions to vulnerable groups. Finally, the review provides input to the conceptualisation and measurements of concepts (i.e. peer influence, availability in schools) which may refine survey instruments and theoretical frameworks concerning eating behaviours.
\end{abstract}

Keywords: fruit, vegetables, child, adolescent, qualitative, review, school, family, availability, eating behaviour

\footnotetext{
* Correspondence: rkr@niph.dk

'National Institute of Public Health, University of Southern Denmark,

Copenhagen, Denmark

Full list of author information is available at the end of the article
} 


\section{Background}

Epidemiological evidence for the health benefits of a diet rich in fruit and vegetables is substantial [1-3]. Despite this fact large proportions of children and adolescents do not meet the World Health Organization goal of a daily intake of at least 400 grams of fruit and vegetables [4-6]. Longitudinal studies suggest that eating behaviour such as fruit and vegetable consumption tracks into adulthood which points at the importance of establishing healthy eating behaviour among children and adolescents [7-9].

To enable the development of relevant, effective fruit and vegetable promoting intervention programs and policies targeting children and adolescents it is important to identify the various factors which may influence their consumption of fruit and vegetables and both qualitative and quantitative studies are needed [10].

Quantitative studies are needed to quantify and rank the importance of determinants for children's fruit and vegetable consumption and for example, to assess sociodemographic variations in these. In the first part of this review the evidence from 98 quantitative studies of fruit and vegetable intake among children and adolescents was analysed [11]. In conclusion, the determinants for high consumption levels of fruit and vegetable supported by the strongest evidence were female gender, low age, high socioeconomic position (SEP), high preferences for fruit and vegetables, high parental intake of fruit and vegetables and high availability/accessibility of fruit and vegetables at home.

Qualitative studies can add to this knowledge in several ways. They provide the opportunity to identify yet unknown factors as the research techniques give room for unprecedented answers as opposed to the highly structured interviews used in surveys. Qualitative studies can thereby contribute to the development of comprehensive survey instruments and generate hypotheses about associations which can be tested in future quantitative studies. Furthermore, qualitative studies can generate a more thorough understanding of fruit and vegetable consumption as they usually aim at reflecting the diversity of views on the studied phenomenon within a given population $[10,12]$. Finally, qualitative methods are a useful tool within formative research aiming at designing effective interventions tailored to a given population's own needs and contextual conditions.

Systematic reviews are important for evidence-based practice. Such review efforts have almost solely been focused on quantitative studies which is also the case for reviews concerning dietary behaviours [10,11,13-15]. It is important also to review the qualitative research to increase insight into processes which influence young people's fruit and vegetable intake. Thus, the aim of the present paper is to present part two of a systematic review of peer-reviewed papers, this time qualitative studies of 6-18-year-olds' views and experiences regarding determinants of their intake of fruit and vegetables.

\section{Methods}

\section{Literature search strategy}

We conducted a comprehensive systematic and exhaustive literature search of the following electronic databases from the year of their inception to December 1st 2010: Anthropology Plus (1900 onwards), CSA illumine [ERIC (1966 onwards), Econlit (1969 onwards), Sociological abstracts (1952 onwards), Social Services abstracts (1979 onwards), Worldwide political Science abstracts (1975 and onwards)], Cinahl (1981 onwards), Embase (1980 onwards), International Bibliography of the Social Sciences (1981 onwards), PsycINFO (1806 onwards), Pubmed Medline (1966 onwards), Web of Science [Science Quotation Index expanded (1945 onwards), Social Sciences Quotation Index (1956 onwards), Art \& Humanities Quotation Index (1945 onwards)]. Together these databases covered a variety of disciplines: anthropology, economics, health education, medicine, nursing research, nutrition, political science, psychology, social care, and sociology.

\section{Search terms}

We applied the same electronic search strategy in all databases. Peer-reviewed publications were searched by combining the following search terms:

1) [(fruit OR fruits OR vegetable OR vegetables) $O R$ ((diet OR diets OR nutrition OR eating OR food) AND (healthy OR healthful))]

\section{AND}

2) [child OR children OR childhood OR adolescent OR adolescents OR adolescence OR youth OR young OR teen OR teens OR teenager OR teenagers OR student OR students OR girl OR girls OR boy OR boys OR pupil OR pupils OR schoolchild OR schoolchildren]

\section{AND}

3) [anthropology OR anthropologic OR anthropological OR ethnography or ethnographic or ethnographical OR qualitative OR focus group OR focus groups OR grounded theory]

The literature search was completed by December 1st 2010 and yielded 2, 813 records. Titles and abstracts were systematically screened and considered for inclusion by one reviewer (RK) according to pre-specified inclusion and exclusion criteria. Papers were included if they 1) investigated determinants of fruit and/or vegetable intake, either as the primary focus or as part of healthy eating (diet, nutrition or food) where information specifically related to fruit and/or vegetables could be identified, 2) explored children's and adolescents' 
own views and experiences of motivators and barriers to eating fruit and vegetables, 3) were based on qualitative research methods (data collection and analysis), and 4) were based on populations within an identifiable agerange of 6 to 18 years (school-aged children). We applied a broad definition of qualitative research methods. Qualitative data collection methods were defined to include observations, face to face interviews, focus group discussions, and action research. Similarly qualitative data analysis was not restricted to certain analytical traditions but defined to include various approaches such as grounded theory, comparative methods, phenomenological analysis, and content analysis.

Papers were excluded if they were 1) based on quantitative research methods (data collection or analysis), 2) review studies, 3) non-peer reviewed or unpublished manuscripts (abstracts or dissertations), and 4) reported in languages other than English.

If the eligibility of a paper was questionable, the paper was provisionally included. Through this screening process 248 potentially relevant papers were identified and the full-text articles were read thoroughly and considered for inclusion by at least two reviewers. If the two authors did not agree upon inclusion, a third author was asked for her opinion, and arguments for eligibility were discussed to come to a joint decision. We also checked our own records, reference lists of all eligible papers as well as bibliographies of existing reviews for relevant papers. Only 24 additional records were identified through these sources, of which one was included in the present review [16].

\section{Analytical approach}

The review of the papers we included followed a 3-step protocol and was based on published recommendations and other systematic reviews of qualitative research as well as the protocol applied in the earlier review from this research group [11,17-20]. We developed standardised templates which were used in each step. All papers were reviewed independently by at least two of the authors (RK and PD/MR). Inter-reviewer disagreement in relation to data extraction was solved by discussion until consensus on interpretation was reached or acknowledged as different perspectives to refine the coding scheme. Two of the three reviewers had conducted qualitative research themselves (RK/PD). All reviewers were involved in the earlier review of quantitative studies (part one) [11] and are researchers within the area of adolescent health and health behaviour.

\section{Step I: Data extraction}

In the first step, we extracted data from each paper into separate paper-specific summary forms on the following topics: 1 . Country setting, 2. Aims, 3. Study population/ sample characteristics, 4 . Study design, 5 . Preconceptions (e.g. theoretical framework), 6. Phenomenon of interest (e.g. fruit and vegetable intake as primary focus or as part of healthy eating), 7. Use of interview guide, 8. Analysis, 9. Main topics (For papers on healthy eating, findings were only extracted if specifically related to fruit and vegetable intake), 10. Main conclusions/discussion, 11. Study limitations, and 12 . Validity issues.

\section{Step II: Quality assessment}

The second analytical step included a systematic assessment of the internal and external validity and overall methodological quality of each paper. We applied a list of quality criteria (see additional file 1 for more information) suggested by different papers on qualitative methodology $[17,21,22]$ and mapped methodological strengths and limitations for each study in a quality assessment scheme. We assessed whether 1) the relevant information on methodological issues/selected criteria was provided in a clear, explicit and sufficient way and if we were able to follow the analysis and the transformation of raw data (interviews) to findings/themes (high communicative validity) 2) whether the quality of craftsmanship in the investigation was high so the findings appeared to be credible, 3) whether the transferability of findings was discussed (high external validity) and 4) if studies could guide future research and practice (pragmatic validity) [23]. At least two of the authors assessed the quality of the papers independently (RK and MR/ $\mathrm{PD})$. Inconsistencies were discussed until consensus was reached. If consensus was hard to obtain the opinion of the third author was sought or a second opinion was obtained from an impartial colleague, a professor experienced in qualitative research methods and working with children as respondents (peer debriefing). We applied two measures for overall quality of the paper, a count of quality criteria met and the evaluators' overall assessment of the quality of the paper.

\section{Step III: Synthesis of findings}

The third step was extraction of all key findings and illustrative quotations into one comprehensive overall evidence scheme. We categorised the findings by constant comparative analysis - a systematic procedure to identify similarities and differences in findings across studies $[17,24]$. Findings from different studies were grouped into the same theme if the analysis showed they shared common characteristics or represented different aspects/dimensions of the same theme. Dissimilar findings were separated out and renamed into other themes. The potential of qualitative research is to depict the scope and variation in children's views and extreme or unusual views mentioned by a few informants might 
represent important properties of a key theme [22]. Therefore findings mentioned only by a few informants or by a few studies were also considered relevant in this review. By this conceptual analysis we de-contextualised our findings in order to identify common themes/potential determinants across countries. To ensure that context was not lost in the synthesis we kept track of the country origin of each extracted key finding and quotation to be able to highlight potential country-differences in the presentation of results. The results from all studies meeting the inclusion criteria were summarized irrespective of methodological quality. As part of sensitivity analysis, papers of high and low methodological quality were compared for congruence and variation in results. We also assessed if different conclusions were reached if the studies of low quality were excluded from the synthesis.

\section{Results}

\section{Study selection and study populations}

Thirty-one papers matched all inclusion criteria and were included in the review. The papers have been published from 1973 to 2009. Most studies were conducted in the United States of America (US, N = 19), followed by Europe $(\mathrm{N}=7)$. Thirteen studies used source triangulation and interviewed both schoolchildren, parents, school staff or key informants from schools or communities. Almost all studies recruited participating children through schools. The majority of studies included general adolescent populations but three studies focused on a selected sample considered to be at greater risk of unhealthy dietary habits such as alternative high school students or diabetic youth. The studies included covered an age range from four to 19 years of age. All studies except one study of boy scouts [25] and four with no information on gender, included both boys and girls. Table 1 summarises the study characteristics.

\section{Evaluation of papers: overall quality assessment}

We evaluated 13 studies to be of high methodological quality and 12 and six studies to be of medium and low methodological quality respectively (based on agreement between at least two of the reviewers). The high quality studies were characterised by a transparent and systematic research process (explicit aims and a clear presentation of preconceptions, sampling, data collection, data analysis, findings, and validation of findings) and included a thorough discussion of transferability of findings, study limitations and contribution of the study to the previous knowledge base. The common characteristics of the studies of low methodological quality (table 2) were insufficient description of aims, preconceptions, data collection and analysis and lack of discussion of study limitations, transferability of findings and the contribution of the study. However, for two of these studies a thorough description of the qualitative study was not the main aim of the paper $[26,27]$.

In addition to the overall assessment of the methodological quality, we also counted the number of quality criteria met out of 63 possible. The studies in the highest third met more than 46 quality criteria (11 assessed as high and 1 medium quality), studies in the medium third met 36-46 criteria ( 2 assessed as high, 6 medium quality and 1 low quality), and studies in the lowest third (5 assessed as medium and 5 low quality) met less than 34 criteria. Maximum number of quality requirements (54 out of 63) were met in the study by MongeRojas et al. (2005) and minimum (19 out of 63) in the study by Cullen et al. (2007) [26,28].

\section{Synthesis of findings from the reviewed studies}

We identified sixteen main themes 1) Preferences/liking and variety, 2) Sensory and physical attributes of fruit and vegetables, 3) Satiety value/hunger satisfaction/filling power, 4) Outcome expectations/expectancies, 5) Knowledge, 6) Food categorisation: perceptions of fruit and vegetables, 7) Fruit and vegetable preparation skills (behavioural capability), 8) Convenience and time costs, 9) Price and affordability, 10) Situational norms: Appropriateness of time, settings/locations and occasions for eating fruit and vegetables, 11) Availability and exposure to fruit and vegetables at home, 12) Availability and exposure to fruit and vegetables in school, 13) Availability and exposure to fruit and vegetables in neighbourhood/local area, and 14) Parental influence, 15) Peer influence, and 16) TV/media influence. The content of each main theme is described below. Within each theme we have highlighted the country origin of the finding if there were inconsistent findings across countries, or if the theme was only discussed in one or a few country settings. No mentioning of study setting in relation to a theme indicates that there were no marked differences. Only age-, gender-, socioeconomic- and ethnic variations in findings which were explicitly mentioned in a paper will be presented in the synthesis below.

\section{Preferences/liking and variety}

Most of the papers do not separate taste, preferences, and taste preferences. We distinguish taste and preferences and define preferences as children's choice of some food items over others and taste as a sensory attribute. Across country settings, it is a consistent finding that taste and preferences are important and that children reported a higher preference for fruit than vegetables [16,25,29-39]. Variety was identified as an important aspect of preferences in two studies from the US and one from New Zealand. Children who liked a broad variety of fruit and vegetables appeared to be more likely to eat ample amount of these foods. The 
Table 1 Study characteristics

\begin{tabular}{|c|c|c|c|c|c|c|c|c|}
\hline $\begin{array}{l}\text { Study by } \\
\text { first } \\
\text { author } \\
\text { [Ref. ID] }\end{array}$ & $\begin{array}{l}\text { Country } \\
\text { setting }\end{array}$ & $\begin{array}{l}\text { Pheno- } \\
\text { menon of } \\
\text { interest } \\
\text { (outcome) }\end{array}$ & $\begin{array}{l}\text { Sampling and } \\
\text { participants }\end{array}$ & $\begin{array}{l}\text { Child socio- } \\
\text { demographics: } \\
\text { A: sex. B: age. } \\
\text { C: school year. D: SEP. } \\
\text { E: ethnic background. }\end{array}$ & $\begin{array}{l}\text { Data collection } \\
\text { methods and no. of FGs } \\
\text { or interviews }\end{array}$ & $\begin{array}{l}\text { Theoretical } \\
\text { framework }\end{array}$ & $\begin{array}{l}\text { Analytical method/ } \\
\text { approach }\end{array}$ & $\begin{array}{l}\text { Main topics related to } \\
\text { FV intake }\end{array}$ \\
\hline $\begin{array}{l}\text { Baranow- } \\
\text { ski et al. } \\
1993[29]\end{array}$ & US & FV intake & $\begin{array}{l}\text { School-based (1 } \\
\text { school): } 235 \\
\text { schoolchildren, } 15 \\
\text { parents, } 8 \text { teachers, } 4 \\
\text { school food service } \\
\text { workers. }\end{array}$ & $\begin{array}{l}\text { A: no info. B: no info. C: } 4 \\
\text { \& 5. D: pre-dominantly } \\
\text { lower SEP. E: more than } \\
50 \% \text { Afro-Americans and } \\
\text { the rest mostly Anglo- } \\
\text { Americans. }\end{array}$ & $\begin{array}{l}\text { FG discussions: } 5 \text { year } 4 \\
\text { schoolchild FGs, } 5 \text { year } 5 \\
\text { schoolchild FGs, } 2 \text { parent } \\
\text { FGs, } 2 \text { teacher FGs, } 1 \\
\text { school food service } \\
\text { worker FG. }\end{array}$ & $\begin{array}{l}\text { Social cognitive } \\
\text { theory: reciprocal } \\
\text { determinism. }\end{array}$ & $\begin{array}{l}\text { No clear description of } \\
\text { analytical procedures. } \\
\text { Theory-based } \\
\text { interpretation of data. } \\
\text { Results are categorised } \\
\text { by aspects of reciprocal } \\
\text { determinism. }\end{array}$ & $\begin{array}{l}\text { Home and school FV } \\
\text { availability, access to } \\
\text { unhealthy food in school, } \\
\text { sensory attributes (taste, } \\
\text { appeal, appearance, smell, } \\
\text { mouth feel), methods of } \\
\text { preparation, preferences/ } \\
\text { liking, outcome } \\
\text { expectancies, acceptance } \\
\text { of national } \\
\text { recommendations, food } \\
\text { categorisation, } \\
\text { preparation skills. }\end{array}$ \\
\hline $\begin{array}{l}\text { Bauer et } \\
\text { al. } 2004 \\
{[57]}\end{array}$ & US & $\begin{array}{l}\text { Healthy } \\
\text { nutrition } \\
\text { (and physical } \\
\text { activity) }\end{array}$ & $\begin{array}{l}\text { School-based (2 } \\
\text { schools): } 26 \\
\text { schoolchildren and } 23 \\
\text { faculty and staff } \\
\text { members. }\end{array}$ & $\begin{array}{l}\text { A: mixed. B: no info. C: } 7 \\
\text { \& } 8 \text {. D (school level): } \\
\text { mixed composition. E: } \\
\text { mixed composition ( } 80 \% \\
\text { White, } 20 \% \text { either Asian- } \\
\text { or African- Americans). }\end{array}$ & $\begin{array}{l}\text { FG discussions: } 7 \text { grade- } \\
\text { and gender- } \\
\text { homogeneous } \\
\text { schoolchild groups, } 3 \\
\text { faculty and staff member } \\
\text { groups and } 10 \text { individual } \\
\text { interviews with key } \\
\text { informants (e.g. school } \\
\text { nurse, cafeteria manager, } \\
\text { administratives). }\end{array}$ & $\begin{array}{l}\text { Ecological models } \\
\text { by Bronfenbrenner } \\
\text { (1979), Stokols } \\
\text { (1996), Story, } \\
\text { Neumark-Stzainer } \\
\text { \& French (2002). }\end{array}$ & $\begin{array}{l}\text { Grounded theory: 1st } \\
\text { step: systematic coding } \\
\text { of themes, 2nd step: } \\
\text { identification of } 3 \\
\text { mechanisms of influences } \\
\text { on eating within the } \\
\text { school environment } \\
\text { based on data-developed } \\
\text { concepts and theoretical } \\
\text { framework. }\end{array}$ & $\begin{array}{l}\text { School FV availability } \\
\text { (quantity, variety, quality), } \\
\text { School availability of } \\
\text { unhealthy competitive } \\
\text { food choices. }\end{array}$ \\
\hline $\begin{array}{l}\text { Booth et } \\
\text { al. } 2008 \\
{[49]}\end{array}$ & Australia & $\begin{array}{l}\text { Healthy food } \\
\text { (Perceived } \\
\text { causes of } \\
\text { overweight } \\
\text { and obesity) }\end{array}$ & $\begin{array}{l}\text { School-based (3 } \\
\text { secondary schools): } \\
58 \text { schoolchildren. }\end{array}$ & $\begin{array}{l}\text { A: mixed, B: 12-17. C: 7- } \\
\text { 11. D (area level): areas } \\
\text { selected to reflect a wide } \\
\text { range of SEP differences. } \\
\text { E: No info. }\end{array}$ & $\begin{array}{l}9 \text { gender- and school } \\
\text { year-homogeneous FGs } \\
\text { (year } 7+8 \text {, year } 9+10 \text {, year } \\
\text { 11). }\end{array}$ & No info. & $\begin{array}{l}\text { No clear description of } \\
\text { analytical procedures. } \\
\text { Coding of themes. }\end{array}$ & $\begin{array}{l}\text { School FV availability } \\
\text { (price, quality, } \\
\text { presentation). }\end{array}$ \\
\hline $\begin{array}{l}\text { Campbell } \\
2009[30]\end{array}$ & US & $\begin{array}{l}\text { Dietary } \\
\text { choices }\end{array}$ & $\begin{array}{l}\text { School-based (1 } \\
\text { school): } 12 \\
\text { schoolchildren. }\end{array}$ & $\begin{array}{l}\text { A: mixed. B: 14-16. C: } 9 \text { \& } \\
\text { 10. D: pre-dominantly } \\
\text { low-income families. E: } \\
\text { mixed (Hispanic, African- } \\
\text { American, Eurasian and } \\
\text { combination of these). }\end{array}$ & $\begin{array}{l}\text { FG discussions: one } \\
\text { group interviewed twice, } \\
\text { during lunch and } \\
\text { immediately after. }\end{array}$ & $\begin{array}{l}\text { Developmental } \\
\text { psychology by } \\
\text { Piaget and Erikson. }\end{array}$ & $\begin{array}{l}\text { Content analysis, but no } \\
\text { clear description of } \\
\text { analytical procedures. }\end{array}$ & $\begin{array}{l}\text { Home and school FV } \\
\text { availability, parental } \\
\text { influence, availability, } \\
\text { liking, methods of } \\
\text { preparation, knowledge, } \\
\text { food categorisation. }\end{array}$ \\
\hline $\begin{array}{l}\text { Cullen et } \\
\text { al. } 1998 \\
{[25]}\end{array}$ & US & $\begin{array}{l}\text { FV con- } \\
\text { sumption }\end{array}$ & $\begin{array}{l}\text { Community-based: } 99 \\
\text { urban boy scouts and } \\
39 \text { parents. }\end{array}$ & $\begin{array}{l}\text { A: boys. B: 10-14. C: } \\
\text { elementary school. D: no } \\
\text { info. E: mainly African- } \\
\text { American (88\%). }\end{array}$ & $\begin{array}{l}13 \text { FGs with boy scouts } \\
\text { and } \\
7 \text { FGs with parents. }\end{array}$ & $\begin{array}{l}\text { Social cognitive } \\
\text { theory concept of } \\
\text { reciprocal } \\
\text { determinism. }\end{array}$ & $\begin{array}{l}\text { No clear description of } \\
\text { analytical procedures. } \\
\text { Transcripts were coded } \\
\text { and quantified. }\end{array}$ & $\begin{array}{l}\text { Preferences, outcome } \\
\text { expectancies, sensory } \\
\text { attributes (taste, mouth } \\
\text { feel), snack food } \\
\text { purchases, price, parental- } \\
\text {, peer-, and media } \\
\text { influence, preparation } \\
\text { skills, home accessibility, } \\
\text { school availability. }\end{array}$ \\
\hline
\end{tabular}


Table 1 Study characteristics (Continued)

\begin{tabular}{|c|c|c|c|c|c|c|c|c|}
\hline $\begin{array}{l}\text { Cullen et } \\
\text { al. } 2000 \\
{[48]}\end{array}$ & US & FJV intake & $\begin{array}{l}\text { School-based (6 } \\
\text { schools): } 180 \\
\text { schoolchildren and } 40 \\
\text { parents. }\end{array}$ & $\begin{array}{l}\text { A: no info. B: 9-12. C: 4-6. } \\
\text { D: mixed. E: mixed: } 3 \\
\text { Afro-American schools, } 1 \\
\text { Euro-American school, } 2 \\
\text { Mexican-American } \\
\text { schools. }\end{array}$ & $\begin{array}{l}\text { School year and } \\
\text { ethnically homogeneous } \\
\text { FG discussions: } 6 \text { African- } \\
\text { American schoolchild FGs, } \\
6 \text { Euro-American } \\
\text { schoolchild FGs, } 5 \\
\text { Mexican-American } \\
\text { schoolchild FGs, and } 8 \\
\text { Parent FGs. }\end{array}$ & $\begin{array}{l}\text { Social cognitive } \\
\text { theory: reciprocal } \\
\text { determinism. }\end{array}$ & $\begin{array}{l}\text { Data-based analysis. } \\
\text { Systematic coding of } \\
\text { transcripts and } \\
\text { comparisons of results by } \\
\text { ethnicity. Data-based } \\
\text { variable names assigned } \\
\text { to text passages. }\end{array}$ & $\begin{array}{l}\text { Home availability/ } \\
\text { accessibility (variety), } \\
\text { peer-, parental-, and } \\
\text { media influence, sensory } \\
\text { attributes (taste), food } \\
\text { categorisation. }\end{array}$ \\
\hline $\begin{array}{l}\text { Cullen et } \\
\text { al. } 2007 \\
{[26]}\end{array}$ & US & School food & $\begin{array}{l}\text { School-based (6 } \\
\text { schools): } \\
\text { schoolchildren, school } \\
\text { staff and district } \\
\text { school food } \\
\text { administrators (no. of } \\
\text { participants not } \\
\text { provided). }\end{array}$ & $\begin{array}{l}\text { A: no info. B: 11-14. C: } \\
\text { middle school. D (school- } \\
\text { level): at least } 50 \% \text { of } \\
\text { schoolchild population } \\
\text { received free or reduced } \\
\text { price meals. E (school- } \\
\text { level): at least } 50 \% \text { of } \\
\text { schoolchild population } \\
\text { was African- American } \\
\text { and Hispanic. }\end{array}$ & $\begin{array}{l}11 \text { FGs with } \\
\text { schoolchildren/school } \\
\text { staff. Interviews with } 7 \\
\text { district school food } \\
\text { administrators. }\end{array}$ & No info. & $\begin{array}{l}\text { No clear description of } \\
\text { analytical procedures. }\end{array}$ & $\begin{array}{l}\text { School V availability } \\
\text { (variety, freshness). }\end{array}$ \\
\hline $\begin{array}{l}\text { Evans et } \\
\text { al. } 2006 \\
\text { [40] }\end{array}$ & US & $\begin{array}{l}\text { Healthful } \\
\text { eating }\end{array}$ & $\begin{array}{l}48 \text { adolescents from } \\
\text { two middle schools } \\
\text { and one recreation \& } \\
\text { parks centre. }\end{array}$ & $\begin{array}{l}\text { A: mixed. B: 10-14. C: } 6 \text { \& } \\
\text { 7. D: low income. E: } \\
\text { Mainly Black (81\%). }\end{array}$ & 3 male and 2 female FGs. & $\begin{array}{l}\text { Social cognitive } \\
\text { theory }\end{array}$ & $\begin{array}{l}\text { Systematic analysis based } \\
\text { on pre-specified coding } \\
\text { scheme (categorisation of } \\
\text { data according to } \\
\text { gender, location, and } \\
\text { motivational theme) and } \\
\text { standardised procedures. }\end{array}$ & $\begin{array}{l}\text { Nutritional knowledge/ } \\
\text { outcome expectations } \\
\text { (misconceptions), home } \\
\text { availability (access to } \\
\text { competitive food } \\
\text { choices), peer pressure/ } \\
\text { symbolic value of food, } \\
\text { school availability } \\
\text { (appearance, appeal, } \\
\text { freshness), FV availability } \\
\text { at restaurants. Gender } \\
\text { differences. }\end{array}$ \\
\hline $\begin{array}{l}\text { Fitzgerald } \\
\text { et al. } 2009 \\
\text { [41] }\end{array}$ & Australia & $\begin{array}{l}\text { Eating } \\
\text { behaviour } \\
\text { (and physical } \\
\text { activity) }\end{array}$ & $\begin{array}{l}\text { School-based (1 } \\
\text { school): } 37 \\
\text { schoolchildren. }\end{array}$ & $\begin{array}{l}\text { A: mixed. B: no info. C: } \\
\text { kindergarten \& year 1-6. } \\
\text { D: low SEP community. E: } \\
\text { no info. }\end{array}$ & $\begin{array}{l}3 \text { FGs: kindergarten }+ \\
\text { year 1-2, year 3-4, year 5- } \\
6 .\end{array}$ & $\begin{array}{l}\text { The socio- } \\
\text { ecological } \\
\text { approach is cited } \\
\text { in the } \\
\text { introduction. }\end{array}$ & $\begin{array}{l}\text { Open coding/thematic } \\
\text { analysis of transcripts. }\end{array}$ & $\begin{array}{l}\text { Outcome expectancies, } \\
\text { adult-, peer- and media } \\
\text { influence, symbolic value } \\
\text { of food, sensory attributes } \\
\text { (taste), convenience, } \\
\text { access to unhealthy food } \\
\text { in local area, time } \\
\text { limitations. }\end{array}$ \\
\hline $\begin{array}{l}\text { Gellar et } \\
\text { al. } 2007 \\
{[31]}\end{array}$ & US & $\begin{array}{l}\text { Healthy } \\
\text { eating }\end{array}$ & $\begin{array}{l}140 \text { youth from } \\
\text { diabetes camp. }\end{array}$ & $\begin{array}{l}\text { A: mixed. B: 7-16 (mean } \\
\text { age: } 11.8) \text {. C: no info. D: } \\
\text { mixed. E: mixed (71\% } \\
\text { white, } 18 \% \text { Black, } 6 \% \\
\text { Hispanic). }\end{array}$ & $\begin{array}{l}12 \text { female and } 6 \text { male } \\
\text { FGs (almost similar age). }\end{array}$ & No info. & $\begin{array}{l}\text { Content analysis: } \\
\text { Systematic coding of } \\
\text { transcripts using a pre- } \\
\text { specified coding system. }\end{array}$ & $\begin{array}{l}\text { Preferences, sensory } \\
\text { attributes (taste), } \\
\text { knowledge, outcome } \\
\text { expectancies, school FV } \\
\text { availability (appeal, } \\
\text { methods of preparation/ } \\
\text { form, competitive } \\
\text { unhealthy food choices), } \\
\text { convenience, home } \\
\text { availability of unhealthy } \\
\text { food, peer- and parental } \\
\text { influence. }\end{array}$ \\
\hline
\end{tabular}


Table 1 Study characteristics (Continued)

\begin{tabular}{|c|c|c|c|c|c|c|c|c|}
\hline $\begin{array}{l}\text { Goh et al. } \\
2009 \text { [53] }\end{array}$ & US & $\begin{array}{l}\text { Healthy } \\
\text { eating (and } \\
\text { physical } \\
\text { activity) }\end{array}$ & $\begin{array}{l}\text { School-based (2 } \\
\text { schools): } 119 \\
\text { schoolchildren, } 63 \\
\text { parents, and } 28 \text { key } \\
\text { stakeholders. }\end{array}$ & $\begin{array}{l}\text { A: mixed. B: mean age: } \\
\text { 12. C: } 7 \text { \& } 8 \text {. D: no info. E: } \\
\text { mixed (58\% Latino). }\end{array}$ & $\begin{array}{l}6 \text { male and } 8 \text { female } \\
\text { schoolchild FGs, } 8 \text { parent } \\
\text { FGs, interviews with } 28 \\
\text { key stakeholders. }\end{array}$ & No info. & $\begin{array}{l}\text { Systematic content } \\
\text { analysis. }\end{array}$ & $\begin{array}{l}\text { School availability } \\
\text { (accessibility, appearance, } \\
\text { methods of preparation, } \\
\text { visibility, braces-friendly } \\
\mathrm{FV} \text {, unhealthy food), } \\
\text { knowledge, parental } \\
\text { influence. }\end{array}$ \\
\hline $\begin{array}{l}\text { Hill et al. } \\
1998[32]\end{array}$ & $\begin{array}{l}\text { New } \\
\text { Zealand }\end{array}$ & $\begin{array}{l}\text { FV con- } \\
\text { sumption }\end{array}$ & $\begin{array}{l}\text { Community-based: } 20 \\
\text { teenagers and their } \\
\text { parent. }\end{array}$ & $\begin{array}{l}\text { A: mixed. B: 13-16. C: no } \\
\text { info. D: mixed. E: Pakeha } \\
\text { (European ancestry). }\end{array}$ & $\begin{array}{l}20 \text { interviews: Separate } \\
\text { interviews with teenager } \\
\text { and parent responsible } \\
\text { for food preparation. }\end{array}$ & No info. & $\begin{array}{l}\text { Cross-household analysis. } \\
\text { The analysis focus on } \\
\text { interaction between } \\
\text { teenager and parent- } \\
\text { shopper in each } \\
\text { household. No clear } \\
\text { description of analytical } \\
\text { procedures. }\end{array}$ & $\begin{array}{l}\text { Situational norms, } \\
\text { convenience, FV } \\
\text { preparation skills, FV } \\
\text { availability at home, } \\
\text { school, and in local area } \\
\text { (appeal, quality, parental } \\
\text { facilitation, price, variety), } \\
\text { peer-, parental- and } \\
\text { media influence, } \\
\text { preferences, outcome } \\
\text { expectancies, knowledge. } \\
\text { Age and gender } \\
\text { differences. }\end{array}$ \\
\hline $\begin{array}{l}\text { Husby et } \\
\text { al. } 2008 \\
{[51]}\end{array}$ & Denmark & $\begin{array}{l}\text { Meals \& } \\
\text { snack } \\
\text { consumption }\end{array}$ & $\begin{array}{l}\text { Children with a } \\
\text { healthy diet }(\mathrm{N}=9) \\
\text { and a less healthy } \\
\text { diet }(\mathrm{N}=8) \text { were } \\
\text { recruited through a } \\
\text { dietary survey among } \\
\text { their parents. }\end{array}$ & $\begin{array}{l}\text { A: mixed. B: 10-11. C: no } \\
\text { info. D: mixed. E: no info. }\end{array}$ & $\begin{array}{l}17 \text { photo-elicited, semi- } \\
\text { structured individual } \\
\text { interviews. }\end{array}$ & $\begin{array}{l}\text { Meals are } \\
\text { examined as social } \\
\text { events. Meals } \\
\text { involve the } \\
\text { establishment and } \\
\text { re-establishment of } \\
\text { the family unit. }\end{array}$ & $\begin{array}{l}\text { Template analysis (pre- } \\
\text { specified themes) and } \\
\text { comparative analysis. }\end{array}$ & $\begin{array}{l}\text { Peer influence (food } \\
\text { swapping), snack, } \\
\text { outcome expectancies, FV } \\
\text { preparation skills, parental } \\
\text { facilitation, food rules. } \\
\text { Gender differences. }\end{array}$ \\
\hline $\begin{array}{l}\text { Keim et } \\
\text { al. } 2001 \\
{[33]}\end{array}$ & US & FV intake & $\begin{array}{l}\text { Community-based: } 27 \\
\text { Caucasian and } 30 \\
\text { Mexican-American } \\
\text { healthy, low income } \\
\text { children from public } \\
\text { school, migrant } \\
\text { worker summer } \\
\text { schools and } \\
\text { community centres. }\end{array}$ & $\begin{array}{l}\text { A: mixed. B: 8-11. C: 3. D: } \\
\text { low income. E: Caucasian- } \\
\text { and Mexican-American. }\end{array}$ & $\begin{array}{l}\text { FG discussions: } 4 \text { FGs of } \\
\text { Caucasian children and } 6 \\
\text { FGs of Mexican-American } \\
\text { children. }\end{array}$ & $\begin{array}{l}\text { Social cognitive } \\
\text { theory }\end{array}$ & $\begin{array}{l}\text { Transcripts analysed and } \\
\text { coded within the context } \\
\text { of Social cognitive } \\
\text { theory. }\end{array}$ & $\begin{array}{l}\text { Parental facilitation, FV } \\
\text { preparation skills, FV } \\
\text { shopping, price, home } \\
\text { availability/accessibility } \\
\text { (visibility, convenience, } \\
\text { variety, unhealthy food), } \\
\text { parental- and peer } \\
\text { influence, preferences, } \\
\text { sensory attributes (taste, } \\
\text { mouth feel, appearance, } \\
\text { quality, freshness, } \\
\text { methods of preparation, } \\
\text { familiarity), outcome } \\
\text { expectancies, knowledge. } \\
\text { Ethnic differences. }\end{array}$ \\
\hline $\begin{array}{l}\text { Khunti et } \\
\text { al. 2008 } \\
\text { [50] }\end{array}$ & UK & $\begin{array}{l}\text { Healthy } \\
\text { lifestyle }\end{array}$ & $\begin{array}{l}\text { School-based: Pupils } \\
\text { (no. not provided but } \\
\text { can be estimated to } \\
\text { maximum 144) and } \\
\text { school staff. }\end{array}$ & $\begin{array}{l}\text { A: mixed. B: } 11-15 . \text { C: } 7- \\
\text { 10. D: schools located in } \\
\text { a very deprived area. E: } \\
\text { mixed: In the overall } \\
\text { sample } 77 \% \text { of the pupils } \\
\text { were of South Asian } \\
\text { origin. }\end{array}$ & $\begin{array}{l}\text { Action research approach. } \\
\text { Baseline: } 18 \text { schoolchild- } \\
\text { and } 5 \text { staff FGs. Follow- } \\
\text { up: } 8 \text { schoolchild- and } 5 \\
\text { staff FGs. Observational } \\
\text { visits at all schools. }\end{array}$ & No info. & $\begin{array}{l}\text { Open coding (in line } \\
\text { with the } 1 \text { st analytical } \\
\text { step of grounded theory) } \\
\text { of data. A process of } \\
\text { progressive focussing is } \\
\text { used to develop a } \\
\text { thematic framework. }\end{array}$ & $\begin{array}{l}\text { Peer influence (image), } \\
\text { cost \& risk of wasting } \\
\text { money, hunger } \\
\text { satisfaction. }\end{array}$ \\
\hline
\end{tabular}


Table 1 Study characteristics (Continued)

\begin{tabular}{|c|c|c|c|c|c|c|c|c|}
\hline $\begin{array}{l}\text { Kim et al. } \\
2007 \text { [42] }\end{array}$ & US & $\begin{array}{l}\text { Dietary } \\
\text { practices/FV } \\
\text { intake (and } \\
\text { physical } \\
\text { activity) }\end{array}$ & $\begin{array}{l}\text { Community-based: } \\
\text { Low-income Hmong } \\
\text { American parents ( } N \\
=44) \text { and youth }(N= \\
\text { 40). Key informants ( } N \\
=5) \text { in Hmong } \\
\text { communities. }\end{array}$ & $\begin{array}{l}\text { A: mixed. B: 11-14. C: no } \\
\text { info. D: low-income. E: } \\
\text { Hmong Americans. }\end{array}$ & $\begin{array}{l}8 \text { FGs with adults and } \\
\text { youths and } 5 \text { individual } \\
\text { interviews with key } \\
\text { informants. }\end{array}$ & No info. & $\begin{array}{l}\text { No clear description of } \\
\text { analytical procedures. The } \\
\text { transcripts were coded } \\
\text { and organised. }\end{array}$ & $\begin{array}{l}\text { Outcome expectancies, } \\
\text { knowledge, preferences, } \\
\text { parental influence, } \\
\text { sensory attributes (smell, } \\
\text { freshness), time/occasions } \\
\text { for eating FV, school } \\
\text { availability. }\end{array}$ \\
\hline $\begin{array}{l}\text { Kirby et } \\
\text { al. } 1995 \\
{[34]}\end{array}$ & US & FV intake & $\begin{array}{l}\text { School-based (6 } \\
\text { schools from } 3 \\
\text { regions): } 398 \\
\text { schoolchildren, } 108 \\
\text { parents, } 43 \text { teachers, } \\
29 \text { school food } \\
\text { service workers. }\end{array}$ & $\begin{array}{l}\text { A: no info. B: no info. C: } \\
4-5 \text {. D (region level): } \\
\text { mixed. E: mixed. } 2 \\
\text { schools of predominantly } \\
\text { white. Caucasian ethnic } \\
\text { composition ( } 1 \text { high and } \\
1 \text { middle SEP) and } 4 \\
\text { schools of non-white } \\
\text { (African-, Asian-, Hispanic } \\
\text { American, other or multi- } \\
\text { ethnic) composition (2 } \\
\text { Low and } 2 \text { very low SEP). }\end{array}$ & $\begin{array}{l}\text { FG discussions: } 15 \\
\text { schoolchild- (school year } \\
\text { homogeneous), } 11 \\
\text { parent-, } 6 \text { teacher- and } 6 \\
\text { Food service worker-FGs. }\end{array}$ & $\begin{array}{l}\text { Social learning } \\
\text { theory: reciprocal } \\
\text { determinism. }\end{array}$ & $\begin{array}{l}\text { Systematic, theory-guided } \\
\text { coding of transcripts. The } \\
\text { assigned variable names } \\
\text { were developed based } \\
\text { on the discussion guide } \\
\text { and theoretical } \\
\text { framework. }\end{array}$ & $\begin{array}{l}\text { Home availability/ } \\
\text { accessibility (variety, } \\
\text { parental facilitation), } \\
\text { preparation skills, price, } \\
\text { preferences (variety liked), } \\
\text { sensory attributes (taste, } \\
\text { mouth feel), food } \\
\text { categorisation, } \\
\text { knowledge, convenience, } \\
\text { methods of preparation, } \\
\text { outcome expectancies, } \\
\text { time/occasions for eating } \\
\text { FV (restaurants), peer } \\
\text { influence, availability in } \\
\text { local area/restaurants. SEP } \\
\text { differences. }\end{array}$ \\
\hline $\begin{array}{l}\text { Kubik et } \\
\text { al. } 2005 \\
{[35]}\end{array}$ & US & $\begin{array}{l}\text { Dietary } \\
\text { practice (and } \\
\text { physical } \\
\text { activity) }\end{array}$ & $\begin{array}{l}\text { School-based (7 } \\
\text { Alternative High } \\
\text { Schools): } 70 \\
\text { schoolchildren. }\end{array}$ & $\begin{array}{l}\text { A: mixed. B: no info. C: 9- } \\
\text { 12. D: (school-level): } \\
\text { mixed composition: } 46 \% \\
\text { of schoolchildren } \\
\text { qualified for free reduced } \\
\text { lunch program. E: mixed } \\
\text { composition: } 36 \% \text { of } \\
\text { schoolchildren were of } \\
\text { non-Caucasian origin } \\
\text { (American-Indian, African- } \\
\text { American, Hispanic, } \\
\text { Asian). }\end{array}$ & 7 schoolchild FGs. & $\begin{array}{l}\text { Ecological theory } \\
\text { and social learning } \\
\text { theory. }\end{array}$ & $\begin{array}{l}\text { Systematic 3-step } \\
\text { analytical process as } \\
\text { described by Miles \& } \\
\text { Huberman (1994). }\end{array}$ & $\begin{array}{l}\text { Convenience, home and } \\
\text { school FV availability/ } \\
\text { accessibility, access to } \\
\text { unhealthy competitive } \\
\text { food in school and local } \\
\text { area, price, quality, } \\
\text { preferences, cooking skills. }\end{array}$ \\
\hline $\begin{array}{l}\text { Lauten- } \\
\text { schlager \& } \\
\text { Smith } \\
2007[36]\end{array}$ & US & $\begin{array}{l}\text { Dietary } \\
\text { behaviour } \\
\text { (values, } \\
\text { beliefs and } \\
\text { gardening \& } \\
\text { cooking } \\
\text { behaviours) }\end{array}$ & $\begin{array}{l}\text { Community-based: } 40 \\
\text { inner-city youth. Two } \\
\text { subgroups: involved } \\
\text { in Youth Farm } \\
\text { Garden Program ( } N= \\
\text { 26) and not involved } \\
(\mathrm{N}=14) \text {. }\end{array}$ & $\begin{array}{l}\text { A: mixed. B: 9-15. C: no } \\
\text { info. D: no info. E: mixed: } \\
\text { white (15\%), African- } \\
\text { American (30\%), Hispanic } \\
(17 \%), \text { Asian (27\%), Somali } \\
\text { (7\%), other or multiracial } \\
(14 \%) \text {. }\end{array}$ & $\begin{array}{l}6 \text { FGs: } 3 \text { with garden } \\
\text { program participants and } \\
3 \text { with youth not } \\
\text { involved in garden } \\
\text { program. }\end{array}$ & $\begin{array}{l}\text { Theory of planned } \\
\text { behaviour }\end{array}$ & $\begin{array}{l}\text { Application of systematic, } \\
\text { content analysis } \\
\text { procedures by Miles \& } \\
\text { Huberman (1994). }\end{array}$ & $\begin{array}{l}\text { Sensory attributes } \\
\text { (flavour/taste, mouth feel/ } \\
\text { texture, appearance) } \\
\text { convenience, preferences, } \\
\text { method of preparation, } \\
\text { outcome expectancies, } \\
\text { knowledge, availability in } \\
\text { the neighbourhood } \\
\text { (seasonality, quality, } \\
\text { quantity, supply), } \\
\text { parental-, peer- and } \\
\text { media influence. }\end{array}$ \\
\hline
\end{tabular}


Table 1 Study characteristics (Continued)

\begin{tabular}{|c|c|c|c|c|c|c|c|c|}
\hline $\begin{array}{l}\text { Libman } \\
2007[43]\end{array}$ & US & $\begin{array}{l}\text { Food } \\
\text { conscious- } \\
\text { ness and } V \\
\text { eating habits }\end{array}$ & $\begin{array}{l}\text { Community-based: } 10 \\
\text { schoolchildren from a } \\
\text { children garden } \\
\text { program, four } \\
\text { mothers and one } \\
\text { father. }\end{array}$ & $\begin{array}{l}\text { A: mixed. B: 10-14. C: no } \\
\text { info. D: no info. E: African- } \\
\text { American, Puerto Rican, } \\
\text { Dominican, and } \\
\text { Guyanese. }\end{array}$ & $\begin{array}{l}\text { Schoolchildren: } 1 \text { FG, } 10 \\
\text { semi-structured seated } \\
\text { interviews, } 6 \text { walking } \\
\text { interviews (youth-led } \\
\text { garden tours). } 5 \text { parent } \\
\text { telephone interviews. } \\
\text { Observations of program } \\
\text { and material. }\end{array}$ & $\begin{array}{l}\text { Developmental } \\
\text { psychology by Lev } \\
\text { Vygotsky. }\end{array}$ & $\begin{array}{l}\text { Systematic coding for } \\
\text { themes relevant to } \\
\text { research questions (Miles } \\
\text { \& Huberman 1994). }\end{array}$ & $\begin{array}{l}\text { Sensory attribute (taste, } \\
\text { methods of preparation), } \\
\text { cooking skills, food } \\
\text { consciousness/ } \\
\text { knowledge, home FV } \\
\text { availability (appearance, } \\
\text { freshness, safety of } \\
\text { organic FV). }\end{array}$ \\
\hline $\begin{array}{l}\text { McKinley } \\
\text { et al. } 2005 \\
{[44]}\end{array}$ & $\begin{array}{l}\text { England and } \\
\text { Northern } \\
\text { Ireland }\end{array}$ & $\begin{array}{l}\text { Healthy } \\
\text { eating }\end{array}$ & $\begin{array}{l}\text { School-based (11 } \\
\text { schools):106 } \\
\text { schoolchildren. }\end{array}$ & $\begin{array}{l}\text { A: Mixed, B: 11-12. C: 1st } \\
\text { year of post-primary } \\
\text { school. D (school level): } \\
\text { mixed SEP backgrounds. } \\
\text { E: mixed ethnic } \\
\text { backgrounds: White } \\
\text { Europeans (76\%), Asian } \\
(18 \%), \text { Afro-Caribbean } \\
(6 \%) .\end{array}$ & $\begin{array}{l}11 \text { FGs ( } 2 \text { discussion } \\
\text { sessions per group). } 4 \text { of } \\
\text { the FGs were gender- } \\
\text { homogeneous as they } \\
\text { were conducted at } \\
\text { single-sex school. }\end{array}$ & No info. & $\begin{array}{l}\text { Systematic coding of } \\
\text { transcripts using the cut- } \\
\text { and-paste technique } \\
\text { described by Stewart \& } \\
\text { Shamdasini (1990). }\end{array}$ & $\begin{array}{l}\text { Food categorisation, } \\
\text { school availability } \\
\text { (appearance, quality), } \\
\text { sensory attributes } \\
\text { (texture, mouth feel), } \\
\text { convenience \& time costs, } \\
\text { cost \& taste guarantee, } \\
\text { cost \& filling power, } \\
\text { rebellion. Gender } \\
\text { differences. }\end{array}$ \\
\hline $\begin{array}{l}\text { Molaison } \\
\text { et al. } 2005 \\
{[37]}\end{array}$ & US & FV intake & $\begin{array}{l}\text { Community-based: } 42 \\
\text { southern, low-income } \\
\text { black American } \\
\text { adolescents recruited } \\
\text { from National Youth } \\
\text { Sport Program. }\end{array}$ & $\begin{array}{l}\text { A: Mixed. B: 10-13. C: no } \\
\text { info. D: low income. E: } \\
\text { Black Americans. }\end{array}$ & $\begin{array}{l}6 \text { gender- and age- } \\
\text { homogeneous FGs. }\end{array}$ & $\begin{array}{l}\text { Social cognitive } \\
\text { theory }\end{array}$ & $\begin{array}{l}\text { Theory guided the } \\
\text { analysis. Transcripts were } \\
\text { coded by content } \\
\text { analysis methods and } \\
\text { codes/themes were } \\
\text { assigned to the } \\
\text { theoretical framework. }\end{array}$ & $\begin{array}{l}\text { Sensory attribute (taste, } \\
\text { method of preparation, } \\
\text { form (canned vs. fresh)), } \\
\text { allergies, preferences, } \\
\text { variety (vegetable } \\
\text { boredom), outcome } \\
\text { expectancies, food } \\
\text { preparation skills, home } \\
\text { and neighbourhood } \\
\text { availability, appropriate } \\
\text { settings for FV, family- } \\
\text { and peer influence, self- } \\
\text { efficacy. Gender } \\
\text { differences. }\end{array}$ \\
\hline $\begin{array}{l}\text { Monge- } \\
\text { Rojas et } \\
\text { al. } 2005 \\
{[28]}\end{array}$ & Costa Rica & $\begin{array}{l}\text { Healthful } \\
\text { eating }\end{array}$ & $\begin{array}{l}\text { School-based (3 } \\
\text { schools): } 108 \\
\text { schoolchildren. }\end{array}$ & $\begin{array}{l}\text { A: mixed. B: 12-18. C: 7- } \\
11 . \mathrm{D} \text { (school-level): } \\
\text { mixed ( } 2 \text { public high } \\
\text { schools and } 1 \text { private } \\
\text { high school). E: Costa } \\
\text { Rican. }\end{array}$ & $\begin{array}{l}12 \text { gender- and age- } \\
\text { homogeneous FGs ( } 3 \\
\text { sessions per group). }\end{array}$ & $\begin{array}{l}\text { Conceptual model } \\
\text { for adolescent } \\
\text { eating behaviours } \\
\text { based on Social } \\
\text { cognitive theory } \\
\text { and ecological } \\
\text { perspective } \\
\text { proposed by Story } \\
\text { et al. (2002). }\end{array}$ & $\begin{array}{l}\text { The transcripts were } \\
\text { reviewed systematically } \\
\text { for emerging themes. } \\
\text { Themes were identified } \\
\text { according to the } \\
\text { theoretical framework. }\end{array}$ & $\begin{array}{l}\text { Knowledge, school } \\
\text { availability of FV and } \\
\text { unhealthy food, home } \\
\text { availability, parental } \\
\text { facilitation, peer } \\
\text { influence/norms (gender } \\
\text { roles, symbolic value of } \\
\text { food), cost \& satiety value, } \\
\text { sensory attributes (taste, } \\
\text { methods of preparation), } \\
\text { convenience \& time } \\
\text { considerations, outcome } \\
\text { expectations, parental- } \\
\text { and media influence. } \\
\text { Gender differences. }\end{array}$ \\
\hline
\end{tabular}


Table 1 Study characteristics (Continued)

\begin{tabular}{|c|c|c|c|c|c|c|c|c|}
\hline $\begin{array}{l}\text { Neumark- } \\
\text { Stzainer } \\
\text { et al. } 1999 \\
{[45]}\end{array}$ & US & $\begin{array}{l}\text { Food } \\
\text { choices and } \\
\text { eating } \\
\text { behaviours }\end{array}$ & $\begin{array}{l}\text { School-based (2 } \\
\text { schools): } 141 \\
\text { schoolchildren. }\end{array}$ & $\begin{array}{l}\text { A: mixed. B: } 12-14 \text { (mean } \\
\text { age: 12.6) \& 15-19 (mean } \\
\text { age: 16.0). C: } 7 \text { \& 10. D: } \\
\text { no info. E: mixed } \\
\text { composition: white (40\%), } \\
\text { Asian-American (25\%), } \\
\text { African-American (21\%), } \\
\text { multiracial (7\%), Hispanic } \\
(6 \%), \text { Native American } \\
(1 \%) .\end{array}$ & $\begin{array}{l}21 \text { age- and gender- } \\
\text { homogeneous FGs. }\end{array}$ & $\begin{array}{l}\text { Social cognitive } \\
\text { theory is included } \\
\text { in the discussion. }\end{array}$ & $\begin{array}{l}\text { Systematic analytical } \\
\text { approach using the } \\
\text { constant comparative } \\
\text { method of grounded } \\
\text { theory. }\end{array}$ & $\begin{array}{l}\text { Sensory attributes (taste, } \\
\text { appeal, appearance, } \\
\text { methods of preparation), } \\
\text { convenience \& time } \\
\text { considerations, hunger } \\
\text { satisfaction \& costs, } \\
\text { availability at home, } \\
\text { school and fast food } \\
\text { restaurants (visibility, } \\
\text { accessibility). Gender } \\
\text { differences. }\end{array}$ \\
\hline $\begin{array}{l}\text { Nicklas et } \\
\text { al. } 1997 \\
\text { [27] }\end{array}$ & US & FV intake & $\begin{array}{l}\text { School-based (4 } \\
\text { schools): } 55 \text { high } \\
\text { school schoolchildren. }\end{array}$ & $\begin{array}{l}\text { A: mixed. B: no info. C: } 9 . \\
\text { D: no info. E: mixed. } \\
\text { Participants drawn from a } \\
\text { student cohort of mainly } \\
\text { Caucasian background } \\
\text { (79\%). The rest are of } \\
\text { Hispanic, African- } \\
\text { American, Asian or Native } \\
\text { American origin. }\end{array}$ & $\begin{array}{l}4 \text { FGs (white male, white } \\
\text { female, black male, black } \\
\text { female) - unclear if FGs } \\
\text { mix schoolchildren from } \\
\text { different schools. }\end{array}$ & $\begin{array}{l}\text { The intervention is } \\
\text { based on the } \\
\text { PRECEDE model of } \\
\text { health education } \\
\text { ( } 6 \text { levels of } \\
\text { behaviour change). }\end{array}$ & $\begin{array}{l}\text { No clear description of } \\
\text { analytical procedures. }\end{array}$ & $\begin{array}{l}\text { Outcome expectancies, } \\
\text { sensory attributes (taste), } \\
\text { inconsistency in taste, } \\
\text { home \& school FV } \\
\text { availability (visibility, } \\
\text { variety, presentation/ } \\
\text { appearance), cost, access } \\
\text { to competitive unhealthy } \\
\text { food in school and local } \\
\text { area. }\end{array}$ \\
\hline $\begin{array}{l}\text { O'Dea } \\
2003[52]\end{array}$ & Australia & $\begin{array}{l}\text { Healthful } \\
\text { food (and } \\
\text { physical } \\
\text { activity) }\end{array}$ & $\begin{array}{l}\text { School-based ( } 34 \\
\text { schools): } 213 \\
\text { schoolchildren and } 38 \\
\text { school principals. }\end{array}$ & $\begin{array}{l}\text { A: mixed. B: 7-17. C: 2-11. } \\
\text { D \& E: a representative } \\
\text { mix of SEP and ethnicity. }\end{array}$ & 38 FGs. & $\begin{array}{l}\text { Theory of planned } \\
\text { behaviour and } \\
\text { social learning } \\
\text { theory. }\end{array}$ & $\begin{array}{l}\text { Content analysis (Miles \& } \\
\text { Huberman), systematic } \\
\text { approach. }\end{array}$ & $\begin{array}{l}\text { Outcome expectancies, } \\
\text { food categorisation, } \\
\text { knowledge, home } \\
\text { availability (unhealthy } \\
\text { competitive food } \\
\text { choices), convenience \& } \\
\text { time costs. }\end{array}$ \\
\hline $\begin{array}{l}\text { Ross } 1995 \\
{[46]}\end{array}$ & Scotland & $\begin{array}{l}\text { Food } \\
\text { choices and } \\
\text { preferences }\end{array}$ & $\begin{array}{l}\text { School-based (one } \\
\text { school): } 46 \\
\text { schoolchildren. }\end{array}$ & $\begin{array}{l}\text { A: mixed. B: 10-12 (mean } \\
\text { age: 11). C: primary } 7 \\
\text { year. D (area): School } \\
\text { situated in catchment } \\
\text { area encompassing all } \\
\text { SEP groups. E: } \\
\text { schoolchildren were } \\
\text { predominantly white } \\
\text { (only a few from ethnic } \\
\text { minority backgrounds). }\end{array}$ & $\begin{array}{l}\text { FG discussions: } 2 \text { male } \\
\text { FGs, } 3 \text { female FGs and } 2 \\
\text { mixed-gender FGs. } \\
\text { Planned observations } \\
\text { during lunch time were } \\
\text { not feasible because of } \\
\text { the fact that lunch } \\
\text { occurred in several sites } \\
\text { simultaneously and only } \\
\text { one researcher being } \\
\text { involved in the project. }\end{array}$ & No info. & $\begin{array}{l}\text { Grounded theory } \\
\text { approach }\end{array}$ & $\begin{array}{l}\text { Sensory attributes (taste, } \\
\text { texture), peer norms/ } \\
\text { influence (food swapping, } \\
\text { socially acceptable food), } \\
\text { affordability. }\end{array}$ \\
\hline $\begin{array}{l}\text { Steven- } \\
\text { son et al. } \\
2007[47]\end{array}$ & Ireland & $\begin{array}{l}\text { Healthy } \\
\text { eating }\end{array}$ & $\begin{array}{l}\text { School-based (no } \\
\text { info. on number of } \\
\text { schools): } 73 \\
\text { adolescents. }\end{array}$ & $\begin{array}{l}\text { A: mixed. B: 12-15. C: } \\
\text { second level schools. D: } \\
\text { mixed. E: no info. }\end{array}$ & $\begin{array}{l}12 \text { age- and gender- } \\
\text { homogeneous FGs. }\end{array}$ & $\begin{array}{l}\text { Socio-ecological } \\
\text { approach. }\end{array}$ & $\begin{array}{l}\text { Systematic coding of } \\
\text { transcripts and deviant } \\
\text { case analysis. }\end{array}$ & $\begin{array}{l}\text { Sensory attributes (taste), } \\
\text { parental influence. }\end{array}$ \\
\hline
\end{tabular}


Table 1 Study characteristics (Continued)

\begin{tabular}{|c|c|c|c|c|c|c|c|c|}
\hline $\begin{array}{l}\text { Walker et } \\
\text { al. } 1973 \\
{[16]}\end{array}$ & US & FV intake & $\begin{array}{l}\text { Schoolchildren and } \\
\text { parents (primarily } \\
\text { mothers). No. not } \\
\text { provided, but can be } \\
\text { estimated to } \\
\text { maximum } 220 \\
\text { participants. }\end{array}$ & $\begin{array}{l}\text { A: mixed. B: 9-12 \& 13-17. } \\
\text { C: elementary and high } \\
\text { school. D: middle and } \\
\text { low income families. E: no } \\
\text { info. }\end{array}$ & $\begin{array}{l}\text { FG discussions (school } \\
\text { year-gender-SEP } \\
\text { homogeneous): } 8 \\
\text { elementary schoolchild } \\
\text { FGs ( } 2 \text { boy- } \& 2 \text { girl-low } \\
\text { income FGs and } 2 \text { boy- } \& \\
2 \text { girl-middle income } \\
\text { FGs), } 8 \text { high school } \\
\text { student FGs, and } 6 \text { parent } \\
\text { groups ( } 3 \text { low and } 3 \\
\text { middle income FGs). }\end{array}$ & $\begin{array}{l}\text { No information. } \\
\text { Study conducted } \\
\text { by social } \\
\text { psychologists. }\end{array}$ & $\begin{array}{l}\text { No clear description of } \\
\text { analytical procedures. }\end{array}$ & $\begin{array}{l}\text { Availability/exposure to } \\
\text { FV at home and in local } \\
\text { area (variety), price, } \\
\text { parental style/attitude, } \\
\text { preferences, sensory } \\
\text { attributes (appearance, } \\
\text { colour, texture, taste, } \\
\text { odour, form, method of } \\
\text { preparation), food } \\
\text { prejudices. }\end{array}$ \\
\hline $\begin{array}{l}\text { Wind et } \\
\text { al. 2005 } \\
\text { [38] }\end{array}$ & $\begin{array}{l}\text { The } \\
\text { Netherlands } \\
\text { and } \\
\text { Belgium- } \\
\text { Flanders }\end{array}$ & FV intake & $\begin{array}{l}\text { School-based: } 3 \\
\text { schools from the } \\
\text { Netherlands, } 60 \\
\text { schoolchildren. } 32 \\
\text { schoolchildren from } \\
\text { Belgium, no. of } \\
\text { schools not provided. }\end{array}$ & $\begin{array}{l}\text { A: mixed. B: 10-11. C: 5-6. } \\
\text { D: no info. E: } \\
\text { Netherlands: In two of } \\
\text { the schools almost all } \\
\text { children were from ethnic } \\
\text { minority groups, in one } \\
\text { school all except one } \\
\text { child had both parents } \\
\text { born in the Netherlands. } \\
\text { Belgium-Flanders: } 4 \\
\text { children had parents born } \\
\text { in a foreign country. }\end{array}$ & $\begin{array}{l}\text { FG discussion: } \\
\text { Netherlands: } 2 \text { boy FGs, } \\
1 \text { girl FG, } 5 \text { mixed gender } \\
\text { FGs. Belgium-Flanders: } 1 \\
\text { boy FG, } 1 \text { girl FG, } 2 \\
\text { mixed gender FGs. }\end{array}$ & $\begin{array}{l}\text { Health belief } \\
\text { model, theory of } \\
\text { planned behaviour, } \\
\text { social ecological } \\
\text { models. }\end{array}$ & $\begin{array}{l}\text { No clear description of } \\
\text { analytical procedures. } \\
\text { Determinants are } \\
\text { analysed separately for } \\
\text { fruit and vegetables. } \\
\text { Determinants classified as } \\
\text { personal, home- or } \\
\text { school environmental } \\
\text { factors. }\end{array}$ & $\begin{array}{l}\text { Outcome expectancies, } \\
\text { food categorisation, } \\
\text { sensory attributes (taste, } \\
\text { appearance, texture), } \\
\text { preferences, knowledge/ } \\
\text { awareness, preparation } \\
\text { skills, situational/social } \\
\text { norms (time/settings for } \\
\text { eating FV), convenience, } \\
\text { home and school } \\
\text { availability/accessibility } \\
\text { (visibility, family rules, } \\
\text { parental facilitation), } \\
\text { unhealthy food shopping, } \\
\text { peer-, parental and } \\
\text { teacher influence. Ethnic } \\
\text { and international } \\
\text { differences. }\end{array}$ \\
\hline $\begin{array}{l}\text { Zeinstra } \\
\text { et al. } 2007 \\
\text { [39] }\end{array}$ & $\begin{array}{l}\text { The } \\
\text { Netherlands }\end{array}$ & $\begin{array}{l}\text { FV } \\
\text { preferences }\end{array}$ & $\begin{array}{l}\text { School-based (1 } \\
\text { school): } \\
\text { Schoolchildren } \\
\text { representing } 3 \\
\text { different stages of } \\
\text { cognitive } \\
\text { development. }\end{array}$ & $\begin{array}{l}\text { A: mixed. B: 4-5 (group } \\
\text { A), 7-8 (group B) and 11- } \\
12 \text { (group C). C: 1st, 4th } \\
\text { and last school year of } \\
\text { primary school. D: no } \\
\text { info. E: no info. }\end{array}$ & $\begin{array}{l}4+4 \text { duo interviews with } \\
\text { group } A \text { and } B \text { and } 4 \text { FGs } \\
\text { with group } C \text {. }\end{array}$ & $\begin{array}{l}\text { Cognitive theory } \\
\text { (Piaget). }\end{array}$ & $\begin{array}{l}\text { Transcripts were coded } \\
\text { systematically using a } \\
\text { coding framework based } \\
\text { on research aims, the } \\
\text { interview guide and } \\
\text { previous findings in the } \\
\text { literature. }\end{array}$ & $\begin{array}{l}\text { Preferences, sensory } \\
\text { attributes (taste, texture, } \\
\text { appearance, methods of } \\
\text { preparation, familiarity, } \\
\text { food categorisation), } \\
\text { outcome expectancies, } \\
\text { appropriate time and } \\
\text { occasions for eating FV. } \\
\text { Age differences. }\end{array}$ \\
\hline
\end{tabular}


Table 2 Quality assessment scheme ( $\mathrm{Y}$ indicates 'yes, information is provided'; NA indicates that the criterion is not applicable or relevant for the study)

\begin{tabular}{|c|c|c|c|c|c|c|c|c|c|c|c|c|c|c|}
\hline & $\begin{array}{l}\text { Baranowski } \\
\text { et al. (1993) }\end{array}$ & $\begin{array}{l}\text { Bauer } \\
\text { et al. } \\
(2004)\end{array}$ & $\begin{array}{l}\text { Booth } \\
\text { et al. } \\
\text { (2008) }\end{array}$ & $\begin{array}{l}\text { Campbell } \\
(2009)\end{array}$ & $\begin{array}{l}\text { Cullen } \\
\text { et al. } \\
\text { (1998) }\end{array}$ & $\begin{array}{l}\text { Cullen et } \\
\text { al. (2000) }\end{array}$ & $\begin{array}{l}\text { Cullen et } \\
\text { al. (2007) }\end{array}$ & $\begin{array}{l}\text { Evans } \\
\text { et al. } \\
\text { (2006) }\end{array}$ & $\begin{array}{l}\text { Fitzgerald } \\
\text { et al. } \\
\text { (2009) }\end{array}$ & $\begin{array}{l}\text { Gellar } \\
\text { et al. } \\
\text { (2007) }\end{array}$ & $\begin{array}{l}\text { Goh } \\
\text { et al. } \\
\text { (2009) }\end{array}$ & $\begin{array}{l}\text { Hill et } \\
\text { al. } \\
\text { (1998) }\end{array}$ & $\begin{array}{l}\text { Husby et } \\
\text { al. (2008) }\end{array}$ & $\begin{array}{l}\text { Keim } \\
\text { et al. } \\
\text { (2001) }\end{array}$ \\
\hline
\end{tabular}

by last name

(1998)

(2006) (2009)

(2007) (2009) (1998)

(2008)

(B-Kh)

Aims

Aims and

research

questions are

explicitly stated

Qualitative

approach
appropriate to

answer research

questions

\section{Preconception}

Explicit

theoretical

framework or

literature review

and/or
beliefs

Information on

Y

Y

how theory is

used (NA if

theoretical

Sampling

procedure

Explicit sampling

strategy of field

sites and/or of

children

strategy: how?

\begin{tabular}{lllllll}
$Y$ & $Y$ & $Y$ & $Y$ & $Y$ & $Y$ \\
\hline & $Y$ & $Y$ & $Y$ & $Y$ & $Y$ \\
\hline
\end{tabular}

Y Y

Y

Y

Y

Recruitment

strategy: by

whom?

Explicit

justification of

sampling

strategy

Sampling

strategy reflects

the study

purpose

$\begin{array}{lllllllllllllllll}Y & Y & Y & Y & Y & Y & Y & Y & Y & Y & Y & Y & Y & Y & Y\end{array}$

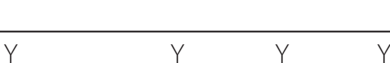

Y

Y

Y $\quad$ Y

$Y$

Y Y

$Y$

Y

Y

$\begin{array}{llllllll}Y & Y & Y & Y & Y & Y & Y\end{array}$

ects

Y NA Y

Y

Y


Table 2 Quality assessment scheme ( $Y$ indicates 'yes, information is provided'; NA indicates that the criterion is not applicable or relevant for the study) (Continued)

\begin{tabular}{|c|c|c|c|c|c|c|c|c|c|c|c|c|c|c|c|}
\hline $\begin{array}{l}\text { Sample size } \\
\text { provided or can } \\
\text { be estimated }\end{array}$ & $Y$ & $Y$ & $Y$ & Y & Y & Y & & Y & $Y$ & Y & Y & $Y$ & $Y$ & $Y$ & $Y$ \\
\hline $\begin{array}{l}\text { Non- } \\
\text { participation } \\
\text { described/ } \\
\text { response rate } \\
\text { (NA if voluntary } \\
\text { sample) }\end{array}$ & & & & NA & Y & Y & & Y & & Y & & & $Y$ & $Y$ & \\
\hline $\begin{array}{l}\text { Sampling/data } \\
\text { collection } \\
\text { continued until } \\
\text { point of data } \\
\text { saturation }\end{array}$ & & & & & & & & & & & Y & & & Y & \\
\hline \multicolumn{16}{|l|}{$\begin{array}{l}\text { Ethical } \\
\text { concerns: } \\
\text { explicit } \\
\text { statement } \\
\text { about...... } \\
\end{array}$} \\
\hline $\begin{array}{l}\text { Informed } \\
\text { consent } \\
\text { (parental or } \\
\text { child) }\end{array}$ & Y & $Y$ & Y & Y & Y & Y & Y & Y & $Y$ & Y & Y & & $Y$ & Y & \\
\hline $\begin{array}{l}\text { Anonymity and } \\
\text { confidentiality }\end{array}$ & & & Y & $Y$ & & & & & & & & & & & $Y$ \\
\hline $\begin{array}{l}\text { Ethical approval/ } \\
\text { review }\end{array}$ & & $Y$ & $Y$ & Y & & Y & Y & $Y$ & $Y$ & Y & $Y$ & & & $Y$ & $Y$ \\
\hline \multicolumn{16}{|l|}{$\begin{array}{l}\text { Sample } \\
\text { characteristics: } \\
\text { Explicit and } \\
\text { sufficient } \\
\text { description } \\
\text { of...... }\end{array}$} \\
\hline $\begin{array}{l}\text { Gender of child } \\
\text { participants }\end{array}$ & & $Y$ & $Y$ & $Y$ & $Y$ & & & $Y$ & Y & Y & $Y$ & $Y$ & $Y$ & $Y$ & $Y$ \\
\hline $\begin{array}{l}\text { Age of child } \\
\text { participants or } \\
\text { school year }\end{array}$ & $Y$ & $Y$ & $Y$ & $Y$ & $Y$ & Y & Y & Y & Y & Y & Y & $Y$ & $Y$ & $Y$ & $Y$ \\
\hline $\begin{array}{l}\text { Socioeconomic } \\
\text { background of } \\
\text { child } \\
\text { participants }\end{array}$ & Y & & & Y & & Y & & Y & & $Y$ & & Y & $Y$ & $Y$ & \\
\hline
\end{tabular}


Table 2 Quality assessment scheme ( $Y$ indicates 'yes, information is provided'; NA indicates that the criterion is not applicable or relevant for the study) (Continued)

\begin{tabular}{|c|c|c|c|c|c|c|c|c|c|c|c|c|c|c|c|}
\hline $\begin{array}{l}\text { Ethnic } \\
\text { background of } \\
\text { child } \\
\text { participants }\end{array}$ & Y & Y & & Y & Y & Y & & Y & & Y & Y & Y & & Y & Y \\
\hline $\begin{array}{l}\text { Other study- } \\
\text { specific } \\
\text { characteristics of } \\
\text { child } \\
\text { participants }\end{array}$ & NA & NA & Y & Y & NA & Y & NA & Y & NA & Y & Y & Y & Y & NA & \\
\hline \multicolumn{16}{|l|}{ Data collection } \\
\hline $\begin{array}{l}\text { Data collection } \\
\text { method (e.g. } \\
\text { focus groups, } \\
\text { observations) } \\
\text { stated }\end{array}$ & Y & Y & Y & Y & Y & Y & Y & Y & Y & Y & Y & Y & Y & Y & Y \\
\hline $\begin{array}{l}\text { Explicit rationale } \\
\text { for data } \\
\text { collection } \\
\text { method } \\
\end{array}$ & Y & Y & & Y & & Y & & Y & Y & Y & & & Y & & \\
\hline $\begin{array}{l}\text { Data collection } \\
\text { methods } \\
\text { adequate to } \\
\text { answer research } \\
\text { questions }\end{array}$ & Y & Y & Y & Y & Y & Y & Y & Y & Y & Y & Y & Y & Y & Y & Y \\
\hline $\begin{array}{l}\text { Number of focus } \\
\text { groups, } \\
\text { interviews, } \\
\text { observations } \\
\text { provided }\end{array}$ & Y & Y & & & Y & Y & Y & Y & Y & Y & Y & & Y & Y & Y \\
\hline $\begin{array}{l}\text { Size of focus } \\
\text { groups } \\
\text { described or } \\
\text { average can be } \\
\text { estimated }\end{array}$ & Y & Y & Y & & Y & Y & & Y & Y & Y & Y & NA & NA & Y & Y \\
\hline $\begin{array}{l}\text { Composition of } \\
\text { child focus } \\
\text { groups/ } \\
\text { interviews } \\
\text { described }\end{array}$ & Y & Y & & & & Y & & Y & Y & Y & Y & & Y & Y & \\
\hline $\begin{array}{l}\text { Explicit rationale } \\
\text { for focus group/ } \\
\text { interview } \\
\text { composition }\end{array}$ & & Y & & & & $\mathrm{Y}$ & & & Y & $Y$ & $Y$ & & NA & Y & \\
\hline $\begin{array}{l}\text { Interview setting } \\
\text { described }\end{array}$ & Y & & Y & Y & Y & Y & & Y & & Y & Y & & Y & Y & \\
\hline
\end{tabular}


Table 2 Quality assessment scheme ( $Y$ indicates 'yes, information is provided'; NA indicates that the criterion is not applicable or relevant for the study) (Continued)

\begin{tabular}{|c|c|c|c|c|c|c|c|c|c|c|c|c|c|c|c|}
\hline $\begin{array}{l}\text { Interviewer } \\
\text { described (who?, } \\
\text { how many?) }\end{array}$ & Y & Y & Y & Y & Y & Y & & Y & Y & Y & Y & & Y & & Y \\
\hline $\begin{array}{l}\text { Duration of } \\
\text { interviews, focus } \\
\text { groups, } \\
\text { observations } \\
\text { described }\end{array}$ & & Y & Y & Y & Y & Y & & Y & & Y & Y & Y & Y & Y & \\
\hline \multicolumn{16}{|l|}{ Interview guide } \\
\hline $\begin{array}{l}\text { Interview guide } \\
\text { used }\end{array}$ & Y & Y & Y & Y & Y & Y & Y & Y & Y & Y & Y & & Y & Y & Y \\
\hline $\begin{array}{l}\text { If yes: Partly } \\
\text { described (key } \\
\text { questions)? Y, } \\
\text { fully described? } \\
\text { YY }\end{array}$ & Y & Y & Y & YY & Y & Y & Y & YY & & YY & YY & & Y & YY & \\
\hline \multicolumn{16}{|l|}{ Analysis } \\
\hline \multicolumn{16}{|l|}{$\begin{array}{l}\text { Reliability/ } \\
\text { consistency }\end{array}$} \\
\hline $\begin{array}{l}\text { Explicit } \\
\text { information of } \\
\text { audiotaping of } \\
\text { interviews }\end{array}$ & & Y & Y & Y & Y & Y & & Y & Y & Y & Y & & Y & Y & \\
\hline $\begin{array}{l}\text { Explicit } \\
\text { information of } \\
\text { transcription of } \\
\text { interviews Y, } \\
\text { verbatim: YY } \\
\end{array}$ & & YY & YY & Y & Y & Y & & YY & Y & YY & Y & Y & YY & Y & \\
\hline \multicolumn{16}{|l|}{$\begin{array}{l}\text { Communicative } \\
\text { validity }\end{array}$} \\
\hline $\begin{array}{l}\text { Analyst } \\
\text { described (who?, } \\
\text { how many?) }\end{array}$ & & Y & Y & & & & & Y & Y & Y & Y & & & Y & \\
\hline $\begin{array}{l}\text { Clear description } \\
\text { of analytical } \\
\text { method? }\end{array}$ & & $Y$ & & & & Y & & Y & Y & Y & Y & & Y & Y & Y \\
\hline $\begin{array}{l}\text { Explicit analytical } \\
\text { approach (data- } \\
\text { based or theory- } \\
\text { based) }\end{array}$ & & Y & & & & Y & & Y & Y & Y & Y & & Y & Y & Y \\
\hline $\begin{array}{l}\text { Analytical } \\
\text { procedures } \\
\text { appropriate to } \\
\text { the research } \\
\text { questions }\end{array}$ & & Y & & & & Y & & Y & Y & Y & Y & & Y & Y & Y \\
\hline
\end{tabular}


Table 2 Quality assessment scheme ( $Y$ indicates 'yes, information is provided'; NA indicates that the criterion is not applicable or relevant for the study) (Continued)

Explicit rationale

for choice of

analytical

procedures

Sampling

strategy/ child

focus group

composition is

used in analysis

Findings/

presentation of

findings

Clear

presentation of

findings

Authors' voices

can always be

distinguished

from informants

voices

Sufficient

inclusion of

quotes to

support findings

Clear description

of selection and

edition of

Different child

participants

views can be

distinguished

The stated
conclusion is

conclusion is
supported by

the findings

Relevance:

Findings/

conclusions

illuminate the

research

questions

Internal validity

Description of

validity and

pilot-testing of

applied

instruments/

guides

\begin{tabular}{|c|c|c|c|c|c|c|c|c|c|c|c|c|}
\hline$Y$ & $Y$ & $Y$ & $Y$ & $Y$ & $Y$ & $Y$ & $Y$ & $Y$ & $Y$ & Y & Y & $Y$ \\
\hline$Y$ & $Y$ & NA & $Y$ & $Y$ & $Y$ & $Y$ & $Y$ & $Y$ & $Y$ & $Y$ & $Y$ & $Y$ \\
\hline
\end{tabular}


Table 2 Quality assessment scheme (Y indicates 'yes, information is provided'; NA indicates that the criterion is not applicable or relevant for the study) (Continued)

Explicit

strategies for

validating

presented

findings

Researcher/

analyst

triangulation

Method

triangulation

Source

triangulation

Theory

triangulation

Peer debriefing/ $Y$

audit trail

Member checks/

respondent

validation

Attention to

negative or

deviant cases

External

validity

Discussion of

(applicability of

findings in othe

contexts)

Explicit

reflections on

selection bias/

non-response of

children

\section{Discussion}

Adequate

attention to

previous

knowledge and

what the study

adds

Findings provide $Y$

new insight on

potential

determinants of

fruit and

vegetables

Y

Y

Y Y

Y

Y $\quad$ Y

Y$$
\text { }
$$ 
Table 2 Quality assessment scheme (Y indicates 'yes, information is provided'; NA indicates that the criterion is not applicable or relevant for the study) (Continued)

\begin{tabular}{|c|c|c|c|c|c|c|c|c|c|c|c|c|c|c|c|c|}
\hline $\begin{array}{l}\text { Discussion of } \\
\text { limitations of } \\
\text { qualitative study }\end{array}$ & Y & Y & & & & Y & & Y & Y & & Y & & & Y & Y & \\
\hline \multicolumn{17}{|l|}{$\begin{array}{l}\text { Pragmatic } \\
\text { validity }\end{array}$} \\
\hline $\begin{array}{l}\text { Discussion of } \\
\text { implications for } \\
\text { research and } \\
\text { practice }\end{array}$ & Y & Y & & Y & Y & Y & Y & Y & Y & Y & Y & Y & Y & Y & & \\
\hline $\begin{array}{l}\text { Quantitative } \\
\text { quality score: } \\
\text { Total number } \\
\text { of quality } \\
\text { requirements } \\
\text { met }\end{array}$ & 32 & 49 & 32 & 32 & 33 & 44 & 19 & 49 & 42 & 46 & 48 & 31 & 43 & 48 & 32 & \\
\hline $\begin{array}{l}\text { Qualitative } \\
\text { quality score } \\
\text { evaluated by } \\
\text { reviewers } \\
\text { (H: high, M: } \\
\text { medium, L: } \\
\text { low) }\end{array}$ & M & $\mathrm{H}$ & L & $L$ & $M$ & $\mathrm{H}$ & L & $\mathrm{H}$ & $M$ & $M$ & $\mathrm{H}$ & $M$ & $M$ & $\mathrm{H}$ & M & \\
\hline $\begin{array}{l}\text { Study listed } \\
\text { alphabetically } \\
\text { by last name } \\
\text { of first author } \\
\text { (Ki-Z) }\end{array}$ & $\begin{array}{l}\text { Kim et al. } \\
\text { (2007) }\end{array}$ & $\begin{array}{l}\text { Kirby } \\
\text { et al. } \\
\text { (1995) }\end{array}$ & $\begin{array}{l}\text { Kubik } \\
\text { et al. } \\
\text { (2005) }\end{array}$ & $\begin{array}{l}\text { Lautenschlager } \\
\text { \& Smith (2007) }\end{array}$ & $\begin{array}{l}\text { Libman } \\
\text { (2009) }\end{array}$ & $\begin{array}{l}\text { McKinley } \\
\text { et al. } \\
\text { (2005) }\end{array}$ & $\begin{array}{l}\text { Molaison } \\
\text { et al. } \\
\text { (2005) }\end{array}$ & $\begin{array}{l}\text { Monge- } \\
\text { Rojas } \\
(2005)\end{array}$ & $\begin{array}{l}\text { Neumark- } \\
\text { Stzainer } \\
\text { et al. } \\
(1999)\end{array}$ & $\begin{array}{l}\text { Nicklas } \\
\text { et al. } \\
\text { (1997) }\end{array}$ & $\begin{array}{l}\text { O'Dea } \\
(2003)\end{array}$ & $\begin{array}{l}\text { Ross } \\
(1995)\end{array}$ & $\begin{array}{l}\text { Stevenson } \\
\text { et al. } \\
\text { (2007) }\end{array}$ & $\begin{array}{l}\text { Walker } \\
\text { et al. } \\
\text { (1973) }\end{array}$ & $\begin{array}{l}\text { Wind } \\
\text { et al. } \\
\text { (2005) }\end{array}$ & $\begin{array}{l}\text { Zeinstra } \\
\text { et al. } \\
\text { (2007) }\end{array}$ \\
\hline \multicolumn{17}{|l|}{ Aims } \\
\hline $\begin{array}{l}\text { Aims and } \\
\text { research } \\
\text { questions are } \\
\text { explicitly stated }\end{array}$ & Y & Y & Y & Y & Y & Y & Y & Y & Y & Y & Y & Y & Y & Y & Y & Y \\
\hline $\begin{array}{l}\text { Qualitative } \\
\text { approach } \\
\text { appropriate to } \\
\text { answer research } \\
\text { questions }\end{array}$ & Y & Y & Y & Y & Y & Y & Y & Y & Y & Y & Y & Y & Y & Y & Y & Y \\
\hline \multicolumn{17}{|l|}{ Preconceptions } \\
\hline $\begin{array}{l}\text { Explicit } \\
\text { theoretical } \\
\text { framework or } \\
\text { literature review } \\
\text { and/or pre-study } \\
\text { beliefs }\end{array}$ & & Y & Y & Y & Y & Y & Y & Y & Y & Y & Y & Y & Y & & Y & Y \\
\hline
\end{tabular}


Table 2 Quality assessment scheme (Y indicates 'yes, information is provided'; NA indicates that the criterion is not applicable or relevant for the study) (Continued)

\begin{tabular}{|c|c|c|c|c|c|c|c|c|c|c|c|c|c|c|c|c|}
\hline $\begin{array}{l}\text { Information on } \\
\text { how theory is } \\
\text { used (NA if no } \\
\text { theoretical } \\
\text { framework) }\end{array}$ & & $Y$ & $Y$ & $Y$ & & NA & $Y$ & $Y$ & $Y$ & $Y$ & $Y$ & NA & & & $Y$ & $Y$ \\
\hline \multicolumn{17}{|l|}{$\begin{array}{l}\text { Sampling } \\
\text { procedure }\end{array}$} \\
\hline $\begin{array}{l}\text { Explicit sampling } \\
\text { strategy of field } \\
\text { sites and/or of } \\
\text { children }\end{array}$ & $Y$ & $Y$ & $Y$ & $Y$ & & $Y$ & $Y$ & $Y$ & $Y$ & & $Y$ & $Y$ & $Y$ & $Y$ & $Y$ & \\
\hline $\begin{array}{l}\text { Recruitment } \\
\text { strategy: how? }\end{array}$ & $Y$ & $Y$ & $Y$ & $Y$ & & $Y$ & $Y$ & $Y$ & $Y$ & $Y$ & $Y$ & $Y$ & $Y$ & & Y & $Y$ \\
\hline $\begin{array}{l}\text { Recruitment } \\
\text { strategy: by } \\
\text { whom? }\end{array}$ & $Y$ & & $Y$ & $Y$ & & & & $Y$ & & Y & $Y$ & $Y$ & $Y$ & & & \\
\hline $\begin{array}{l}\text { Explicit } \\
\text { justification of } \\
\text { sampling } \\
\text { strategy }\end{array}$ & & $Y$ & $Y$ & $Y$ & & $Y$ & $Y$ & Y & $Y$ & & $Y$ & Y & $Y$ & $Y$ & $Y$ & $Y$ \\
\hline $\begin{array}{l}\text { Sampling } \\
\text { strategy reflects } \\
\text { the study } \\
\text { purpose }\end{array}$ & Y & Y & $Y$ & $Y$ & $Y$ & $Y$ & $Y$ & $Y$ & $Y$ & & Y & Y & $Y$ & Y & Y & $Y$ \\
\hline $\begin{array}{l}\text { Sample size } \\
\text { provided or can } \\
\text { be estimated }\end{array}$ & $Y$ & $Y$ & $Y$ & $Y$ & $Y$ & $Y$ & $Y$ & $Y$ & $Y$ & $Y$ & $Y$ & $Y$ & $Y$ & & $Y$ & $Y$ \\
\hline $\begin{array}{l}\text { Non- } \\
\text { participation } \\
\text { described/ } \\
\text { response rate } \\
\text { (NA if voluntary } \\
\text { sample) }\end{array}$ & NA & $Y$ & $Y$ & & $Y$ & & & $Y$ & $Y$ & & $Y$ & $Y$ & & $Y$ & & \\
\hline $\begin{array}{l}\text { Sampling/data } \\
\text { collection } \\
\text { continued until } \\
\text { point of data } \\
\text { saturation }\end{array}$ & & & & $Y$ & & & & & & & & & & & & \\
\hline \multicolumn{17}{|l|}{$\begin{array}{l}\text { Ethical } \\
\text { concerns: } \\
\text { explicit } \\
\text { statement } \\
\text { about...... }\end{array}$} \\
\hline $\begin{array}{l}\text { Informed } \\
\text { consent } \\
\text { (parental or } \\
\text { child) }\end{array}$ & & $Y$ & $Y$ & $Y$ & & $Y$ & $Y$ & $Y$ & $Y$ & & $Y$ & & $Y$ & & $Y$ & $Y$ \\
\hline
\end{tabular}


Table 2 Quality assessment scheme ( $Y$ indicates 'yes, information is provided'; NA indicates that the criterion is not applicable or relevant for the study) (Continued)

\begin{tabular}{|c|c|c|c|c|c|c|c|c|c|c|c|c|c|c|c|c|}
\hline $\begin{array}{l}\text { Anonymity and } \\
\text { confidentiality }\end{array}$ & & & & & & Y & Y & & & & & & Y & & Y & Y \\
\hline $\begin{array}{l}\text { Ethical approval/ } \\
\text { review }\end{array}$ & Y & Y & Y & Y & & Y & Y & Y & & & & & & & Y & \\
\hline \multicolumn{17}{|l|}{$\begin{array}{l}\text { Sample } \\
\text { characteristics: } \\
\text { Explicit and } \\
\text { sufficient } \\
\text { description } \\
\text { of..... }\end{array}$} \\
\hline $\begin{array}{l}\text { Gender of child } \\
\text { participants }\end{array}$ & Y & & Y & Y & Y & Y & Y & Y & Y & Y & Y & Y & Y & Y & Y & Y \\
\hline $\begin{array}{l}\text { Age of child } \\
\text { participants or } \\
\text { school year }\end{array}$ & Y & Y & Y & Y & Y & Y & Y & Y & Y & Y & Y & Y & Y & Y & Y & Y \\
\hline $\begin{array}{l}\text { Socioeconomic } \\
\text { background of } \\
\text { child } \\
\text { participants }\end{array}$ & Y & & & & & & Y & & & & Y & & Y & Y & & \\
\hline $\begin{array}{l}\text { Ethnic } \\
\text { background of } \\
\text { child } \\
\text { participants }\end{array}$ & $Y$ & & Y & $Y$ & Y & $Y$ & Y & $Y$ & $Y$ & Y & $Y$ & Y & & & $Y$ & \\
\hline $\begin{array}{l}\text { Other study- } \\
\text { specific } \\
\text { characteristics of } \\
\text { child } \\
\text { participants }\end{array}$ & NA & Y & Y & Y & & Y & NA & Y & NA & NA & NA & NA & Y & Y & Y & NA \\
\hline \multicolumn{17}{|l|}{ Data collection } \\
\hline $\begin{array}{l}\text { Data collection } \\
\text { method (e.g. } \\
\text { focus groups, } \\
\text { observations) } \\
\text { stated }\end{array}$ & Y & Y & Y & Y & Y & Y & Y & Y & Y & Y & Y & Y & Y & Y & Y & Y \\
\hline $\begin{array}{l}\text { Explicit rationale } \\
\text { for data } \\
\text { collection } \\
\text { method } \\
\end{array}$ & & Y & Y & & Y & Y & & Y & Y & Y & & Y & Y & Y & Y & Y \\
\hline $\begin{array}{l}\text { Data collection } \\
\text { methods } \\
\text { adequate to } \\
\text { answer research } \\
\text { questions }\end{array}$ & Y & $Y$ & $Y$ & $Y$ & $Y$ & Y & Y & $Y$ & Y & Y & $Y$ & $Y$ & Y & $Y$ & $Y$ & Y \\
\hline
\end{tabular}


Table 2 Quality assessment scheme (Y indicates 'yes, information is provided'; NA indicates that the criterion is not applicable or relevant for the study) (Continued)

\begin{tabular}{|c|c|c|c|c|c|c|c|c|c|c|c|c|c|c|c|c|}
\hline $\begin{array}{l}\text { Number of focus } \\
\text { groups, } \\
\text { interviews, } \\
\text { observations } \\
\text { provided }\end{array}$ & Y & Y & Y & Y & Y & Y & Y & Y & Y & Y & Y & Y & Y & Y & Y & Y \\
\hline $\begin{array}{l}\text { Size of focus } \\
\text { groups } \\
\text { described or } \\
\text { average can be } \\
\text { estimated }\end{array}$ & & Y & Y & Y & & Y & Y & Y & Y & Y & & Y & Y & Y & Y & Y \\
\hline $\begin{array}{l}\text { Composition of } \\
\text { child focus } \\
\text { groups/ } \\
\text { interviews } \\
\text { described }\end{array}$ & & Y & Y & Y & & & Y & Y & Y & Y & & Y & Y & Y & Y & Y \\
\hline $\begin{array}{l}\text { Explicit rationale } \\
\text { for focus group/ } \\
\text { interview } \\
\text { composition }\end{array}$ & & & & Y & & & & Y & & & & & Y & & & Y \\
\hline $\begin{array}{l}\text { Interview setting } \\
\text { described }\end{array}$ & & Y & Y & & Y & Y & Y & Y & Y & & & Y & Y & & & Y \\
\hline $\begin{array}{l}\text { Interviewer } \\
\text { described (who?, } \\
\text { how many?) }\end{array}$ & Y & Y & Y & $Y$ & & $Y$ & Y & Y & Y & Y & & Y & Y & Y & Y & Y \\
\hline $\begin{array}{l}\text { Duration of } \\
\text { interviews, focus } \\
\text { groups, } \\
\text { observations } \\
\text { described }\end{array}$ & Y & & Y & Y & Y & Y & & Y & Y & & Y & Y & Y & Y & Y & Y \\
\hline \multicolumn{17}{|l|}{ Interview guide } \\
\hline $\begin{array}{l}\text { Interview guide } \\
\text { used }\end{array}$ & Y & $Y$ & Y & $Y$ & $Y$ & $Y$ & $Y$ & $Y$ & $Y$ & $Y$ & $Y$ & Y & $Y$ & $Y$ & $Y$ & $Y$ \\
\hline $\begin{array}{l}\text { If yes: Partly } \\
\text { described (key } \\
\text { questions)? Y, } \\
\text { fully described? } \\
\text { YY }\end{array}$ & & YY & Y & $Y Y$ & Y & YY & YY & Y & Y & Y & Y & YY & Y & Y & Y & YY \\
\hline \multicolumn{17}{|l|}{ Analysis } \\
\hline \multicolumn{17}{|l|}{$\begin{array}{l}\text { Reliability/ } \\
\text { consistency }\end{array}$} \\
\hline $\begin{array}{l}\text { Explicit } \\
\text { information of } \\
\text { audiotaping of } \\
\text { interviews }\end{array}$ & Y & Y & Y & Y & Y & Y & Y & Y & Y & Y & Y & Y & Y & Y & Y & Y \\
\hline
\end{tabular}


Table 2 Quality assessment scheme (Y indicates 'yes, information is provided'; NA indicates that the criterion is not applicable or relevant for the study) (Continued)

\begin{tabular}{|c|c|c|c|c|c|c|c|c|c|c|c|c|c|c|c|c|}
\hline $\begin{array}{l}\text { Explicit } \\
\text { information of } \\
\text { transcription of } \\
\text { interviews } Y, \\
\text { verbatim: } Y Y\end{array}$ & $Y$ & $Y Y$ & $Y Y$ & $Y Y$ & $Y$ & $Y Y$ & $Y Y$ & $Y Y$ & $Y Y$ & & $Y Y$ & $Y Y$ & $Y$ & & $Y$ & $Y$ \\
\hline \multicolumn{17}{|l|}{$\begin{array}{l}\text { Communicative } \\
\text { validity }\end{array}$} \\
\hline $\begin{array}{l}\text { Analyst } \\
\text { described (who?, } \\
\text { how many?) }\end{array}$ & & $Y$ & $Y$ & $Y$ & & $Y$ & $Y$ & Y & $Y$ & & & $Y$ & & $Y$ & $Y$ & $Y$ \\
\hline $\begin{array}{l}\text { Clear description } \\
\text { of analytical } \\
\text { method? }\end{array}$ & & $Y$ & $Y$ & $Y$ & $Y$ & $Y$ & $Y$ & $\bar{Y}$ & $Y$ & & $Y$ & $Y$ & Y & & & $\bar{Y}$ \\
\hline $\begin{array}{l}\text { Explicit analytical } \\
\text { approach (data- } \\
\text { based or theory- } \\
\text { based) }\end{array}$ & & $Y$ & $Y$ & $Y$ & $Y$ & $Y$ & $Y$ & $\bar{Y}$ & $Y$ & & $Y$ & $Y$ & $Y$ & & $Y$ & $\bar{Y}$ \\
\hline $\begin{array}{l}\text { Analytical } \\
\text { procedures } \\
\text { appropriate to } \\
\text { the research } \\
\text { questions }\end{array}$ & & $Y$ & $Y$ & $Y$ & $Y$ & $Y$ & $Y$ & $Y$ & $Y$ & & $Y$ & $Y$ & $Y$ & & $Y$ & $Y$ \\
\hline $\begin{array}{l}\text { Explicit rationale } \\
\text { for choice of } \\
\text { analytical } \\
\text { procedures }\end{array}$ & & $Y$ & $Y$ & & & $Y$ & $Y$ & $Y$ & $Y$ & & & & $Y$ & $Y$ & $Y$ & $Y$ \\
\hline $\begin{array}{l}\text { Sampling } \\
\text { strategy/ child } \\
\text { focus group } \\
\text { composition is } \\
\text { used in analysis }\end{array}$ & & $Y$ & $Y$ & $Y$ & & $Y$ & $Y$ & $Y$ & $Y$ & $Y$ & $Y$ & & $Y$ & $Y$ & $Y$ & $\bar{Y}$ \\
\hline \multicolumn{17}{|l|}{$\begin{array}{l}\text { Findings/ } \\
\text { presentation of } \\
\text { findings }\end{array}$} \\
\hline $\begin{array}{l}\text { Clear } \\
\text { presentation of } \\
\text { findings }\end{array}$ & $Y$ & $Y$ & $Y$ & $Y$ & $Y$ & $Y$ & $Y$ & $Y$ & $Y$ & $Y$ & $Y$ & $Y$ & $Y$ & $Y$ & $Y$ & $\bar{Y}$ \\
\hline $\begin{array}{l}\text { Authors' voices } \\
\text { can always be } \\
\text { distinguished } \\
\text { from informants' } \\
\text { voices }\end{array}$ & Y & $Y$ & $Y$ & $Y$ & $Y$ & $Y$ & $Y$ & $Y$ & $Y$ & $Y$ & $Y$ & $Y$ & $Y$ & $Y$ & $\mathrm{Y}$ & $\bar{Y}$ \\
\hline
\end{tabular}


Table 2 Quality assessment scheme ( $Y$ indicates 'yes, information is provided'; NA indicates that the criterion is not applicable or relevant for the study) (Continued)

\begin{tabular}{|c|c|c|c|c|c|c|c|c|c|c|c|c|c|c|c|c|}
\hline $\begin{array}{l}\text { Sufficient } \\
\text { inclusion of } \\
\text { quotes to } \\
\text { support findings }\end{array}$ & & & Y & Y & Y & Y & Y & Y & Y & Y & Y & Y & Y & & Y & \\
\hline $\begin{array}{l}\text { Clear description } \\
\text { of selection and } \\
\text { edition of } \\
\text { quotes }\end{array}$ & & & & Y & & & & Y & Y & & & & & & & \\
\hline $\begin{array}{l}\text { Different child } \\
\text { participants' } \\
\text { views can be } \\
\text { distinguished }\end{array}$ & & & Y & Y & & & & Y & Y & & Y & Y & Y & & & \\
\hline $\begin{array}{l}\text { The stated } \\
\text { conclusion is } \\
\text { supported by } \\
\text { the findings }\end{array}$ & Y & Y & Y & Y & Y & Y & Y & Y & Y & Y & Y & Y & Y & Y & Y & Y \\
\hline $\begin{array}{l}\text { Relevance: } \\
\text { Findings/ } \\
\text { conclusions } \\
\text { illuminate the } \\
\text { research } \\
\text { questions }\end{array}$ & Y & Y & Y & Y & Y & Y & Y & Y & Y & Y & Y & Y & Y & Y & Y & Y \\
\hline \multicolumn{17}{|l|}{ Internal validity } \\
\hline $\begin{array}{l}\text { Description of } \\
\text { validity and } \\
\text { pilot-testing of } \\
\text { applied } \\
\text { instruments/ } \\
\text { guides }\end{array}$ & & Y & Y & & Y & Y & Y & Y & Y & & & Y & Y & & Y & Y \\
\hline \multicolumn{17}{|l|}{$\begin{array}{l}\text { Explicit } \\
\text { strategies for } \\
\text { validating } \\
\text { presented } \\
\text { findings }\end{array}$} \\
\hline $\begin{array}{l}\text { Researcher/ } \\
\text { analyst } \\
\text { triangulation }\end{array}$ & & & Y & Y & & Y & Y & Y & Y & & & & & & Y & Y \\
\hline $\begin{array}{l}\text { Method } \\
\text { triangulation }\end{array}$ & Y & & & & Y & & & & & Y & & Y & & & & Y \\
\hline $\begin{array}{l}\text { Source } \\
\text { triangulation }\end{array}$ & Y & Y & & & Y & & & & & & Y & & & Y & & \\
\hline \multicolumn{17}{|l|}{$\begin{array}{l}\text { Theory } \\
\text { triangulation }\end{array}$} \\
\hline $\begin{array}{l}\text { Peer debriefing/ } \\
\text { audit trail }\end{array}$ & & & & & Y & & & Y & Y & & & & & Y & $Y$ & Y \\
\hline
\end{tabular}


Table 2 Quality assessment scheme (Y indicates 'yes, information is provided'; NA indicates that the criterion is not applicable or relevant for the study) (Continued)

\begin{tabular}{|c|c|c|c|c|c|c|c|c|c|c|c|c|c|c|c|c|}
\hline \multicolumn{17}{|l|}{$\begin{array}{l}\text { Member checks/ } \\
\text { respondent } \\
\text { validation }\end{array}$} \\
\hline $\begin{array}{l}\text { Attention to } \\
\text { negative or } \\
\text { deviant cases }\end{array}$ & & & & & & & & & & & & & Y & & & \\
\hline \multicolumn{17}{|l|}{$\begin{array}{l}\text { External } \\
\text { validity }\end{array}$} \\
\hline $\begin{array}{l}\text { Discussion of } \\
\text { transferability } \\
\text { (applicability of } \\
\text { findings in other } \\
\text { contexts) }\end{array}$ & Y & Y & Y & & Y & & Y & Y & & Y & & Y & & & Y & Y \\
\hline $\begin{array}{l}\text { Explicit } \\
\text { reflections on } \\
\text { selection bias/ } \\
\text { non-response of } \\
\text { children }\end{array}$ & Y & & & & Y & & & Y & Y & & & & & & & \\
\hline \multicolumn{17}{|l|}{ Discussion } \\
\hline $\begin{array}{l}\text { Adequate } \\
\text { attention to } \\
\text { previous } \\
\text { knowledge and } \\
\text { what the study } \\
\text { adds }\end{array}$ & & & Y & $Y$ & & Y & Y & Y & Y & & Y & $Y$ & $Y$ & & Y & Y \\
\hline $\begin{array}{l}\text { Findings provide } \\
\text { new insight on } \\
\text { potential } \\
\text { determinants of } \\
\text { fruit and } \\
\text { vegetables }\end{array}$ & Y & Y & Y & $Y$ & Y & Y & Y & Y & Y & $Y$ & $Y$ & Y & Y & Y & Y & Y \\
\hline $\begin{array}{l}\text { Discussion of } \\
\text { limitations of } \\
\text { qualitative study } \\
\end{array}$ & Y & Y & Y & & Y & Y & Y & Y & Y & Y & & Y & & Y & Y & Y \\
\hline \multicolumn{17}{|l|}{$\begin{array}{l}\text { Pragmatic } \\
\text { validity }\end{array}$} \\
\hline $\begin{array}{l}\text { Discussion of } \\
\text { implications for } \\
\text { research and } \\
\text { practice }\end{array}$ & Y & Y & Y & Y & Y & Y & Y & Y & Y & Y & Y & Y & Y & Y & Y & Y \\
\hline
\end{tabular}


Table 2 Quality assessment scheme ( $Y$ indicates 'yes, information is provided'; NA indicates that the criterion is not applicable or relevant for the study) (Continued)

\begin{tabular}{|c|c|c|c|c|c|c|c|c|c|c|c|c|c|c|c|c|}
\hline $\begin{array}{l}\text { Quantitative } \\
\text { quality score: } \\
\text { Total number } \\
\text { of quality } \\
\text { requirements } \\
\text { met }\end{array}$ & 33 & 44 & 50 & 47 & 36 & 47 & 48 & 54 & 50 & 32 & 40 & 47 & 45 & 33 & 46 & 47 \\
\hline $\begin{array}{l}\text { Qualitative } \\
\text { quality score } \\
\text { evaluated by } \\
\text { reviewers } \\
\text { (H: high, M: } \\
\text { medium, L: } \\
\text { low) }\end{array}$ & L & $\mathrm{H}$ & $\mathrm{H}$ & $\mathrm{H}$ & $M$ & $\mathrm{H}$ & $\mathrm{H}$ & $\mathrm{H}$ & $\mathrm{H}$ & $\mathbf{L}$ & L & $M$ & $\mathbf{M}$ & $M$ & $M$ & $\mathrm{H}$ \\
\hline
\end{tabular}


number of fruit and vegetables liked seemed to differ according to SEP and age [25,32,34].

\section{Sensory and physical attributes of fruit and vegetables}

Several studies from a range of countries highlighted sensory and physical attributes of fruit and vegetables as both promoters and barriers for fruit and vegetable consumption among children $[16,25,27-29,31,33,34,36-47]$. The sensory attributes of vegetables were often linked to negative connotations. In one study from the Netherlands, the sensory attributes children considered when evaluating whether they liked or disliked fruit and vegetables differed by age which may reflect different stages of cognitive development [39].

Taste/flavour Many studies concluded that taste is a main reason for not liking fruit and vegetables - especially vegetables $[25,27,29,33,34,36,37,39,41,46]$. Children reported that competitive unhealthy food choices such as sweets and junk foods tasted better $[25,34,38,45,48]$. However, taste also emerged as a positive outcome expectancy of eating especially fruit in two studies from the US [34,37]. Generally, children appeared to prefer the taste of fruit to vegetables because of the sweet flavour [16,29, 32-35,37,38]. Vegetables were associated with unpleasant and negative taste experiences e.g. bitter, sour, taste of nothing, bland, dull, tart, too strong $[16,28,31,33,34,36,37,39$, 44,47]. Familiarity of taste and earlier exposure to fruit and vegetables were found to play an important role in children's acceptance of fruit and vegetables in three studies from the US $[16,33,36]$. Walker et al. (1973) reported that children's distaste for some fruit and vegetables often was more based on prejudice than taste [16]. In another US study, children from youth gardening programmes said that growing and learning more about vegetables made them less prejudiced towards tasting unfamiliar vegetables [43].

The liking and disliking of vegetables was related to methods of preparation, in five studies from the US e.g. many schoolchildren did not like cooked vegetables and home prepared vegetables were preferred to those received in school $[29,30,34,37,43]$. Addition of a topping/condiment or another food to enhance the flavour of fruit and especially vegetables was mentioned as a facilitator for fruit and vegetable consumption in a variety of countries $[28,29,33,36,39,45]$.

Texture and mouth feel The texture and mouth feel of fruit and vegetables was reported as another important factor influencing children's fruit and vegetable consumption across country settings. Whereas fruits were perceived as sweet, juicy and fun to eat, vegetables were often linked to negative sensory experiences, e.g. some children expressed that the texture of some vegetables made them feel sick $[34,39,46]$. Positive aspects of texture that were mentioned were crispy, crunchy and juicy which contributed to children's preference for fresh, raw fruit and vegetables. Negative aspects of texture that were reported across studies were mushy, squishy, slimy, dry, cold, smooshy, gooey, icky, "feels like spittle", too hard, containing seeds, yucky, nasty and related to children's dislike for vegetables, especially cooked vegetables $[16,25,29,33,36,38,39,44,46]$.

Food aesthetics (appearance, sight, presentation, appeal) Stood out as crucial for the children's choice of fruit and vegetables in studies from different country settings $[16,29,32,33,38,43-45,49]$. Negative aspects of appearance were unripe, mouldy, rotten, wrinkled, "looks horrible", "looks weird", ugly, soggy, and boring. Across studies, children aged 10-18 rejected bruised or imperfect fruit such as brown spots as this was interpreted as possible signs of unsatisfactory taste and texture $[16,29,36,40,44-46]$. Appearance seemed to be a more important reason for disliking and rejecting vegetables for younger than older children $[32,39]$.

Colour was an aspect of appearance mentioned by a couple of US studies. Diversity of colours encouraged children to eat more fruit and vegetables [29]. Children linked colours of fruit to favourable taste expectations whereas they perceived the colour of certain vegetables as unappetizing [16].

Smell/odour In three US studies, a pleasant smell also played a role in the children's fruit and vegetable consumption $[16,29,42]$.

\section{Satiety value/hunger satisfaction/filling power}

In several studies across geographical settings, children expressed a concern for the satiety value of fruit and vegetables $[28,44,45,50]$. Children did not think fruit and vegetables were as filling as foods like crisps and chocolate [44]. Another study reported that filling power was an immediate positive outcome expectancy of eating fruit and vegetables [29]. Hunger satisfaction may be a special concern for boys [44].

\section{Outcome expectations/expectancies}

Outcome expectancies were discussed in several studies from a variety of countries, and could be grouped into positive and negative short and long term expectancies.

Short term Across studies, children were able to list positive short term outcome expectancies of eating fruit and vegetables: General health, growth (give you strength, make you stronger, help the body grow and make you taller, more muscular), nutritious and hunger satisfaction (contain vitamins, specific nutrients, good for the eyes and teeth, make you feel full after eating it), cosmetics benefits (looking good, improved body image, improved skin appearance, avoid fatness, remain slender, loose or gain weight, pretty teeth), improve performance and productivity in school work and sports/athletics (fuel for the brain, give energy, refreshing and reviving effect as opposed to unhealthy food that slows down the 
mind and body), sensory aspects (taste, sweetness, crunchiness, fun to eat) $[25,27,29,31-34,36-39,41$, $42,45,51,52]$. Negative short term expectancies of eating fruit and vegetables were explicitly discussed in one US study: Make you go to the loo, gas in your stomach, get stuck in your teeth, allergy [29]. In two US studies some of the schoolchildren expressed a concern about the fat and sugar content in vegetables and fruit and the risk of getting hyper and diabetes when eating it $[33,40]$.

Long term One US study reported that children could mention only a few long term outcome expectations (all positive) of eating fruit and vegetables [29]. Three studies from Costa Rica, US and the United Kingdom (UK) suggested that children saw the health damages of not eating fruit and vegetables as a distant concern for adulthood $[28,45,50]$. Children appear to base their food choices rather on taste, hunger satisfaction, appearance of food, and peer pressure [50]. Other studies reported that children valued the long term outcome expectations (positive expectancy) such as future health as an important reason for eating healthy food $[27,29,36,52]$.

Four studies from the US, New Zealand and Costa Rica suggested that outcome expectancies differed by gender, with boys possibly more concerned with long term disease prevention and general health/fitness aspects and girls with weight loss and weight control $[28,32,40,45]$.

\section{Knowledge}

Dietary/nutritional knowledge Studies from the US and Costa Rica showed that children knew fruit and vegetables were good for them but they were not specific on the relevance to health $[28,36,53]$. Some children were able to list specific healthy effects $[31,33,36,52]$. Hill et al. (1998) reported that dietary knowledge increased by age in New Zealand [32]. Two US studies found that schoolchildren from high SEP neighbourhoods [34] and children who participated in youth gardening programmes could list more specific health benefits of eating fruit and vegetables [36].

Knowledge, awareness and acceptability of national recommendations of fruit and vegetable consumption Four studies included discussion on recommendations. Wind et al. (2005) found low awareness of national recommendations for vegetable consumption among children in the Netherlands and Belgium-Flanders, and many children thought they ate enough fruit and vegetables although hardly any of them ate them every day [38]. According to Campbell et al. (2009) the US schoolchildren in their study knew the recommendations but did not distinguish between fruit and vegetables [30]. In a study among Hmong Americans, children appeared to be more familiar with the definition of a "serving" of fruit and vegetables and the recommendation to eat at least 5 servings of fruit and vegetables daily than their parents [42]. '5-a-day' was perceived as an unreasonably high goal by schoolchildren in the US study by Baranowski et al. (1993) [29].

\section{Food categorisation: Perceptions of fruit and vegetables}

Ten studies from the US, Australia, the UK, Ireland and the Netherlands examined how children categorise and perceive fruit and vegetables $[29,30,34,38,39,44,46-48$, 52]. Two studies reported that children's perceptions of healthy eating almost invariably included fruit, vegetables and salads $[44,52]$. Some studies found that children did not classify their food into healthy and unhealthy, rather into liked and disliked [46,47]. Two studies found that children reasoned that if a food tastes bad it must be good for you and the reverse $[29,47]$. In one study, children (7-12 years old) perceived fruit and vegetables as being appropriate for both children and adults [39] while other studies showed that year 4 and 5 pupils perceived vegetables as being food for grown-ups [34,38]. Some children showed difficulties in deciding which food items belong to the fruit and vegetable group e.g. some children thought that candies containing fruit, soft drinks, lemonade, fruit yoghurt, milk shakes, fruit-flavoured beverages and fruit tea could be defined as fruit or fruit juice or that chips that are based on corn or potatoes could be defined as vegetables [29,30,38]. Other studies found that children did distinguish between fruit and vegetables or perceived salad and vegetables as belonging to different food groups $[30,48]$.

\section{Fruit and vegetable preparation skills (behavioural capability)}

If children are responsible for preparing their own fruit and vegetable snacks they must be able to peel, cut, chop, grate, core or cook fruit and vegetables. Some US studies suggest that children have limited fruit and vegetable preparation skills and are unsure how to prepare and cook vegetable dishes $[25,34]$. A study of primarily low SEP children suggested that all children took part in some kind of food preparation [29]. Kirby et al. (1995) found that almost all pupils participated in preparing their own snacks for home or school. Other studies demonstrated sociodemographic variations in preparation skills [32-34,37], e.g. that US children from low SEP sites were responsible for preparing more meals alone than children from middle or high SEP sites [34], that US girls were involved in complex food preparation tasks whereas boys only assisted in simple food preparation task, if any [37], and that older teenagers and children from New Zealand who were living in a one-parent household more often cooked meals on a regular basis than younger teenagers and children living with two parents [32].

There may be national variations in the extent to which parents allow their children to prepare meals. Wind et al. (2005) found that 11-year-olds from the Netherlands were allowed to and liked to prepare fruit 
and vegetable dishes whereas 11-year-olds from Belgium-Flanders often were not allowed to cut up vegetables themselves or to be involved in preparing the dinner, but that they were allowed to prepare fruit [38]. Similarly some Danish 11-year-olds were not allowed to use sharp knives which prevented them from having pineapples for example if their parents did not cut them up for them [51]. According to Kubik et al. (2005), cooking classes to learn about healthy eating and how to cook cheap food were popular among students in secondary school [54].

\section{Convenience and time costs}

Convenience can be defined as ease of obtaining, preparing, transporting and/or eating fruit and vegetables [45]. Regardless of country setting, lack of convenience emerged as a key barrier to eating fruit and vegetables in several papers $[28,31-36,41,44,45,52]$. Children were not willing to sacrifice time to eat fruit and vegetables, even when liked $[28,32,35,44]$. When choosing and purchasing snack food they had a preference for pre-packaged food that was easy to get, to carry and requiring no preparation such as salty snacks, sweets, fast food, and soft drinks $[33,42,52]$. Fruit and vegetables were in general perceived as inconvenient snack food as they were not instantly available and had to be washed, dried, peeled or cooked before consumed $[28,31,32,35,45]$. Also fruit and vegetables were inconvenient to transport compared to snacks e.g. fruit gets bruised in your back pack on the way to school $[32,38]$. However, one study among 13-16-year-olds from New Zealand deviated from this finding and reported that some children saw fruit as a convenient snack food as it did not require preparation as opposed to vegetables. These children also expressed that they expected their parents to cut up fruit for them for snacks [32].

Time appears to be crucial and children accordingly reported to make a trade off between eating healthily and time. Convenience food was preferred in order to be able to sleep longer and to avoid wasting school breaks waiting in a queue $[28,35,44,45]$. In the Dutch/ Belgian-Flemish study by Wind et al. (2005), some participants said they ate fruit during the weekends more often than on school days, because they had more time [38]. Others had less fruit over the weekends because they were too busy doing other things and did not remember to eat fruit.

\section{Price and affordability}

The importance of affordability was highlighted in ten studies that indicated that price influenced children's purchases and selection of fruit and vegetables in all country settings $[25,27,28,32-35,44-46,49,50]$. The study by Hill et al. (1998) from New Zealand suggested that children's primary shopping criterion was quantity, i.e. to get as much as possible for their money [32]. Other studies from the US and the UK showed that children were also concerned with quality, satiety value and getting value for money $[25,44,50]$. Children would not risk wasting money on new food choices or on fruit and vegetables for which there was no guarantee of a pleasant taste as opposed to a chocolate bar which would always taste the same $[27,44,50]$.

In some studies, children said that a trade off between healthy food and hunger satisfaction motivated their food purchase $[28,45,50]$. They did not think they got value for money if they spent their money on fruit as the price was too high and fruit did not satisfy their hunger. Instead they bought unhealthy food like chips, pastries and fast food that was less expensive and more likely to fill them up. Hill et al. (1998) reported that 1316 year-olds expected their parents to purchase and provide fruit for them, they would not think of buying it with their own money [32].

Some studies suggested that there were socioeconomic and ethnic variations in the importance of affordability for purchase and consumption of fruit and vegetables $[33,34]$. In the US, Kirby et al. (1995) found that fruit and vegetables were only perceived as expensive in the lower SES groups [34]. In a study of low income children more Mexican-American children than Caucasian children reported cost as a barrier to fruit and vegetable purchase [33].

\section{Situational norms: Appropriateness of time, settings/} locations and occasions for eating fruit and vegetables

Children's perceptions of appropriate time, settings and occasions for eating fruit and vegetables influence their opportunities for eating fruit and vegetables.

Time In four studies children stated that dinner was the only appropriate time for eating vegetables $[32,34,38,39]$ which also meant that not having vegetables for dinner was perceived as an important reason for not eating vegetables at all [38]. Fruit, on the other hand was perceived as appropriate to eat at all times of the day in two Dutch studies [38,39]. Zeinstra et al. (2007) noted some age differences e.g. 4-5 year-olds thought that you could only eat fruit for lunch and in the afternoon, whereas 11-12 year-olds thought that you could eat it whenever you felt like eating it [39]. In three studies fruit was usually eaten as a snack $[32,34,42]$. In only one study from the Netherlands and Belgium-Flanders a few participants mainly from minority groups, said they sometimes ate vegetables (a piece of cucumber, carrot or a tomato) as a snack in between meals [38].

Setting/location/occasions Eating fruit was mainly associated with the home environment [39]. In one study children did not perceive time with friends or TVwatching as appropriate occasions for eating fruit and vegetables and reported they would eat more fruit and vegetables if they could eat it while being together with 
friends or watching TV [42]. The children in the study by Molaison et al. (2005) mentioned both home, other family members' homes, school and restaurants as locations for eating fruit and vegetables [37]. However, in another study children from all SEP groups agreed that eating out was a special treat and therefore not a time for eating fruit and vegetables [34]. Eating fruit in school was perceived as normal by Dutch and Flemish adolescents whereas eating vegetables in school was perceived as unusual - although some ethnic differences were noted [38].

Dutch and Flemish 11-12-year-olds in two studies reported that fruit was too healthy to serve at birthdays and other celebrations and that the social norm was to eat sweets on such occasions [38,39]. 4-5 year-olds on the other hand reported that fruit and vegetables could be served at parties [39].

\section{Availability/exposure to fruit and vegetables at home}

Availability of fruit and vegetables at home emerged as an important factor affecting fruit and vegetable consumption in 15 studies from different countries [16,27-30,32-35,37,38,43,45,48,52]. Home availability may reflect socioeconomic and international differences. One example is that vegetables were often not available in Dutch homes whereas they were present in BelgianFlemish homes [38]. Another example is that children in low income families reported that fruit and vegetables were not available at home [33,37].

Variety Variety was suggested as an important aspect of home availability in studies from the US. Two studies reported that children who were exposed to a wide variety of fruit and vegetables at home, liked and ate a greater variety of fruit and vegetables [16,33]. Lack of variety in the fruit and vegetables available was mentioned as a barrier to fruit and vegetable consumption by children in two studies $[27,37]$. In one study all child groups expressed having access to a variety of fruit and vegetables at home [48] whereas one study suggested that children in homes in areas of middle to high SEP had access to a larger variety of fresh fruit and vegetables than children from low SEP areas [34]. Furthermore the parents from low SEP regions included canned and frozen fruit and vegetables more often in the family meals [34].

Home accessibility, parental facilitation and visibility Accessibility means whether fruit and vegetables are available in a form and location that make them convenient to eat [55]. The process, where parents increase home accessibility such as by cutting up fruit for their children to eat in between meals is sometimes referred to as parental facilitation [56]. Low accessibility emerged as a key barrier to fruit and vegetable consumption from the US, Denmark, New Zealand and Costa Rica. Fruit and vegetables seemed to be available in most homes but not easily accessible or visible to the children $[25,33,48,51]$.

Children perceived purchase and preparation of fruit and vegetables as an adult task $[28,32,33]$ and reported that they would eat healthier if their parents and adults in school purchased and provided them with healthy food and encouraged them to eat it by cutting up fruit and vegetables or displaying it by having a bowl of fruit on the table $[35,42,45]$.

Two US studies imply that there are socioeconomic and ethnic differences in children's access to fruit and vegetables and parental facilitation [33,34]. For example in one study children from middle and high SEP sites said that their parents cut up fruit and vegetables for them as snacks and left them within easy reach whereas children from low SEP sites were more responsible for preparing their own meals [34].

Food rules The effect of high availability may be limited by the fact that some children are not allowed to eat as much fruit and vegetables as they like or have to ask before they eat fruit and vegetables. For example Belgium-Flanders children often had to ask their parents whether they could take fruit whereas Dutch participants were more likely to be allowed to take fruit themselves whenever they wanted [38]. In a Danish study schoolchildren said that there were no restrictions on consumption of fruit at home whereas they had to ask for permission to take unhealthy food items [51].

Home availability of unhealthy competitive food choices In four studies from the US and Australia, some children discussed availability of unhealthy, competitive food options (visual cues) at home as a barrier to eating fruit and vegetables [31,33,40,52].

\section{Availability and exposure to fruit and vegetables in school}

Irrespective of country setting, it is a consistent finding across most studies that fruit and vegetables are only available in small quantities in school or not available at all $[25-32,35,38,44,46,57]$.

Variety and choice Children from the US and New Zealand reported they would eat more fruit and vegetables if school offered a larger variety that matched their preferences and served them fresh $[26,27,32,57]$. Some children said that the choice of fruit in school was limited to canned fruit salad which they did not like or, as for the diabetic children in the study by Gellar et al. (2007) they could not eat it because it was "drenched in sugary syrup" $[27,31,32]$.

Quality, appearance and methods of preparation The children in most studies complained about the quality and appearance of the fruit and vegetables available in school. The fruit items available were often bruised, brown, old-looking or of poor quality e.g. mushy $[27,32,35,40,44,45,49]$. The vegetables offered at school 
were cold, mushy, soggy, dry, looked nasty, had an unpleasant taste and were prepared in a non-appealing manner $[29,31,44,45,57]$. The children in three US studies preferred the way vegetables were prepared at home to the way they were prepared at school $[29,34]$ although some children also thought their parents prepared vegetables in an unappetizing manner [48].

Accessibility, visibility, convenience, time costs, and affordability Regardless of country setting low accessibility in school was highlighted as a barrier to eating fruit and vegetables. Many pupils preferred salad bars, bite-sized or sliced fruit instead of unpeeled or whole fruit [53]. Many children complained about vegetables/ salads not being as visible (the children had to ask for them to get them) or promoted as much (e.g. no signs) as unhealthy food items in the school canteen $[27,45]$. Time was also highlighted as a barrier for example children did not want to waste their precious, but short lunch break waiting in a long queue for healthy food $[45,57]$ and they thought that they had too little time for eating at break time [41]. Some children brought fruit and vegetables from home but not all ate it as transportation ruined the appearance of fruit and vegetables $[32,38]$. Some pupils thought they would purchase more fruit and vegetables if it was easily accessible in refrigerated vending machines at school [35]. Further, the high price of fruit and vegetables in school was highlighted as a barrier for eating fruit and vegetables in school in studies from Scotland and Australia $[46,49]$.

Access to competitive unhealthy food choices Another barrier to eating fruit and vegetables in school mentioned by children from the US and Costa Rica was the constant and extensive exposure to unhealthy food in the school environment and the lack of access to affordable healthy food options [28,29,31,35,45,53,57].

\section{Availability and exposure to fruit and vegetables in} neighbourhood/local area

Children in a study from the US said that fruit and vegetables were not available in the grocery stores where their parents purchased food [37]. Seasonality emerged as a barrier to availability of fruit and vegetables in the local area in a study among youth in Minnesota who experienced low access to fresh fruit of high quality in the winter [36]. In two studies children reported that fruit sold nearby was not attractive $[32,36]$. In three US studies children discussed low availability, variety, visibility, and attractiveness of fruit and vegetables in (fast-food) restaurants as a barrier for intake $[34,40,45]$. Children only liked eating fruit and vegetables at restaurants with salad bars they could chose their own fruit and vegetables from [34].

Again high access to competitive unhealthy food choices emerged as a barrier for fruit and vegetable intake as it appeared to be much more convenient and inexpensive for the children to buy unhealthy foods (fast-food) in the local area than fruit $[27,32,34,35,41,45]$.

\section{Parental influence}

Some children learned how to eat fruit and vegetables from a family member and found it easier to eat healthily if the entire family did so $[31,33]$ or if they experienced positive social support and motivation from adults to eat healthily $[25,30,35-37,37,38,42]$. However, in one UK study 11-12-year-olds expressed that they did not like being preached to about dietary habits by their parents and as a consequence ate unhealthily to express independence or rebellion [44]. Some studies from the US and Costa Rica also mentioned parents as social influences for eating unhealthy food e.g. by taking the children to fast food restaurants or by having unhealthy eating habits themselves $[28,36,48,53]$. However, in one US study boy scouts said they were encouraged by their parents to eat fruit and vegetables at restaurants [25].

Hill et al. (1998) found that the family, especially the mother is the primary source of nutritional knowledge among children in New Zealand. In the same study and in a study from Ireland, children said that their parents conveyed mixed messages to them. On the one hand parents tried to limit children's intake of sweets, chocolates, soft drinks and potato crisps, and on the other hand they used the very same unhealthy food items as treats for being good [32,47].

\section{Peer influence}

Peer influences and peer pressure is a challenging issue in focus group discussions with adolescents. Group dynamics and peer pressure may hinder children from speaking freely about it and also the children may not be conscious of how much they are influenced by their peers $[34,36,37,48]$. Keim et al. (2001) suggests that in the US, children are very aware of peers' eating behaviours as almost all children in their study knew how much fruit and vegetables their friends ate [33]. In general, across country settings and age groups, peer influences were not perceived as supporting fruit and vegetable consumption, first and foremost because there was a strong peer pressure towards eating unhealthy food $[28,31,32,34,37,38,40,46]$. Wind et al. (2005) suggest that peers only influence fruit and vegetable consumption among 10-11-year-olds indirectly through promoting higher intake of unhealthy competitive food choices [38], while five studies which covered older age-groups (9-18 years) implied a direct influence as children reported negative comments or being bullied if they brought healthy lunchboxes to school or ate fruit and vegetables in school $[25,28,32,40,48,50]$. Children did not perceive eating fruit in school as 'cool' behaviour although they ate it at home $[41,50]$. 
A few US studies reported positive peer influences towards eating fruit and vegetables and negative peer influences towards eating unhealthy food: Two studies reported that fruit was perceived as acceptable to eat in school but that there was a negative peer influence on eating vegetables in school $[25,48]$. One study found that children who participated in a youth gardening programme did not perceive any peer pressure towards eating unhealthy food [36]. Some of the diabetic children in the study by Gellar et al. (2007) pointed to their peers as supporting them in eating healthily by encouraging them to avoid unhealthy food to control their blood sugar [31].

Positive and negative peer influences towards eating fruit and vegetables may differ by gender because of the symbolic value of eating fruit and vegetable with respect to image and gender identity [28]. Costa Rican boys were considered effeminate by their peers if they ate healthy food and consequently made it a rule to eat unhealthy food items to prove masculinity and bravery. Among girls, eating healthy foods was considered as a sign of femininity and consequently they often would bring cut up fruit to school. Girls risked being bullied if they did not eat healthily [28]. Also, a study from the US is concerned with the symbolic value of food as a barrier for intake: A girl reported being teased about eating as elderly people because of her high intake of fruit and vegetables which suggests that fruit and vegetable consumption symbolizes old age to some children [40].

Peer interaction may also increase access to unhealthy food in schools [31,46]. Food choice in the playground appeared to be determined by socially acceptable food such as sweets, chocolate, carbonated drinks and crisps rather than fruit and vegetables $[41,46]$. Two studies from Denmark and Scotland suggested that girls especially valued the social aspects of meals and snacks [46,51]. In the study by Husby et al. (2009) girls often engaged in swapping food with their friends such as exchanging pizza for grapes [51]. Children in other studies said that nobody wanted to swap healthy food such as fruit and they emphasised the importance of bringing food to school that peers wanted to swap to be part of this shared social event $[31,46]$.

\section{6. $T V /$ media influence}

Most children had not seen any commercials for fruit and vegetables so media influences were only discussed in relation to promotion of unhealthy competitive food choices. Commercials for unhealthy food and fast food was discussed as a barrier to fruit and vegetable consumption as they triggered a craving towards tasty and inexpensive unhealthy food $[25,28,32,36,41,48]$. Media influence was not discussed in any of the European studies.

\section{Sensitivity analysis: results}

The potential determinants identified in the low quality studies were generally in agreement with the ones from the high quality studies e.g. home and school availability, convenience, parental modelling and price. However, one contradictory finding was observed: Two low quality studies $[27,52]$ did not observe any gender differences in outcome expectancies, whereas three high quality studies did [28,40,45]. If focus groups mix girls and boys there might be a risk of false agreement due to peer pressure which may conceal gender differences. Further, lack of a gender focus or non-systematic research processes may have hidden gender differences.

The qualitative studies of low quality did contribute with interesting ideas to the synthesis of findings across studies, even though their non-transparent and less reflective research process made it hard to evaluate the credibility of the findings in these studies. For example the study by Kim et al. (2007) adds to the other studies by showing variations in the identified themes appropriate occasions for eating fruit and vegetables (Hmong youth do not perceive being together with friends or TV-watching as appropriate occasions for eating fruit and vegetables) and availability of fruit and vegetables in school (There is a disagreement between the fruit and vegetables children prefer, and what kind of fruit and vegetables are served in school) [42]. Further, two low quality studies indicated that children would eat more if the school increased the variety of fruit and vegetables offered in school [26,27]. Variety was not identified as a potential determinant in any of the middle or high quality studies. Nicklas et al. (1997) was the first qualitative study to focus on high access to unhealthy competitive food choices as a barrier to fruit and vegetable consumption. The study further pointed at visibility as an important dimension of school availability and inconsistency in taste as an important reason for purchasing chocolate, instead of fruit and vegetables [27]. These two themes were not highlighted in other qualitative studies until 2005, where they appeared as influencing factors in the study by McKinley et al. 2005 [44].

If the review had included only the high quality papers most of the key themes would have emerged. However, the themes 'fruit and vegetable preparation skills (behavioural capability)', 'price and affordability', 'parental influence', 'peer influence' and 'knowledge about recommendations for fruit and vegetable intake' had only been superficially described (unsaturated, thin description). Further, the themes 'food rules' and 'variety of fruit and vegetables in school', 'colour and smell of fruit and vegetables' had not emerged as important potential determinants. We had also not captured the range in children's views e.g. for 'satiety value' and 'outcome expectancies'. The study by Baranowski et al. (1993) (quality assessment: 
medium) added some important dimensions by showing that some children perceived the filling power of fruit as a positive outcome expectancy and that children also associated negative outcome expectancies like for instance allergic reactions and gas build up with eating fruit and vegetables [29]. The high quality studies only highlighted filling power of fruit and vegetables as a barrier to intake $[28,44,45]$.

\section{Discussion}

Thirty-one papers were included in this review and in accordance with earlier reviews of quantitative observational studies $[11,13,15,58,59]$ the present review supports the importance of sociodemographic factors (gender, age/school year, SEP, ethnic background), availability/accessibility of fruit and vegetables at home, parental influences and taste preferences for children's intake of fruit and vegetables. The present synthesis of the results of qualitative studies adds to and enriches the findings in reviews of quantitative studies in five ways (table 3 ):

First of all the present review has identified some potential determinants for fruit and vegetable intake among adolescents which have only been investigated sparsely in quantitative epidemiologic studies, if at all. These factors are summarized in point one of table 3 .

Secondly, the qualitative findings enrich the epidemiologic findings in part one of this review and help us understand the variation, scope and implication of the identified determinants. The review of quantitative studies showed that only one out of five studies on outcome expectations found an association between outcome expectations and children's fruit and vegetable intake $[11,60]$. The qualitative studies suggested that perceived outcome expectations and health benefits of eating fruit and vegetables were not the main concern of children when making food choices rather other aspects such as taste, convenience and sensory appeal mattered.

Further, the review of qualitative studies suggests that cost, appearance, visibility and variety are important dimensions in home availability. None of these dimensions was investigated separately in the studies included in the review of quantitative studies but rather as components of summary scales of 'perceived barriers'. Scales of perceived barriers should be decomposed to investigate the relative importance of items.

The epidemiological evidence from observational studies for an association between availability of fruit and vegetable in schools and pupils' fruit and vegetable consumption is limited $[11,58]$. Meanwhile a review of intervention studies implies that school fruit and vegetable programmes are effective in increasing fruit and vegetable intake among pupils [61]. The present review of qualitative studies suggests that other aspects of school availability than the mere presence of fruit and vegetables matter such as variety, visibility, quality, texture, cost, convenience, time, access to competitive

\section{Table 3 What this review adds}

1. Potential determinants for children's fruit and vegetable intake:

Time costs: trade-off between time and being healthy

Satiating power: fruit and vegetables are perceived as less filling than fast food

Situational norms: perceptions of appropriateness of time, occasions and settings for eating fruit and vegetables

- Important aspects of availability: variety, visibility, methods of preparation, quality of fruit and

vegetables, access to unhealthy food

- Other important sensory aspects than taste: appearance, smell, texture

- Price and inconsistency in taste of fruit and vegetables in comparison with unhealthy food

- Peer influences: sharing food as a means of socialising, the symbolic value of food for image and gender

identity

- School availability: the importance of variety and being able to make your own food choice,

too short breaks for eating fruit and vegetables

- Short term outcome expectancies more important than long term outcome expectations:

Children see long term outcomes as a distant concern of adulthood, they value the immediate benefits or

drawbacks of eating fruit and vegetables

2. Extensive information about potential determinants that have only been sparsely investigated in quantitative studies e.g. peer influence, school availability and thereby new input for conceptualisation and operationalisation of these factors

3. Potential mechanisms behind the observed epidemiological associations (or lack of) between personal, social and environmental factors and children's fruit and vegetable intake such as gender and SEP differences e.g. children from high SEP families are exposed to a larger variety of fruit and vegetables at home and thereby may develop a higher preference for a variety of fruit and vegetables which increases their consumption

4. Potential reasons for children's higher intake of fruit compared to vegetables e.g. they perceive fewer time points, occasions and settings as appropriate for eating vegetables than fruit

5. Awareness about the shortage of qualitative studies within this research area from other countries than US 
unhealthy food and methods of preparation. The quantitative studies have focused on parental intake of fruit and vegetables as a promoter of children's own intake [11]. The qualitative studies suggest that it may be worth studying parents' intake of unhealthy food as a barrier for healthy eating habits among children.

The third contribution of the present review of qualitative research is that it points at new hypotheses which need to be tested quantitatively. The qualitative evidence suggests that gender roles, peer pressure, the symbolic value of food and different expectancies of the outcomes of eating fruit and vegetables as possible explanations for why girls have a higher or more frequent intake of fruit and vegetables than boys.

Further, a potential chain of causation behind the welldocumented social inequality in children's fruit and vegetables intake is suggested. Children from high income families are exposed to a larger variety of fruit and vegetables and as a consequence may develop preferences for a larger variety of fruit and vegetables resulting in a higher intake of fruit and vegetables compared to children from families of low SEP [16,34].

Most epidemiological studies find that young adolescents have a higher or more frequent intake of fruit and vegetables than older adolescents [11]. One possible reason for this suggested by our review is that when children grow older, the extent of parental support for eating fruit and vegetables decreases. Parents expect the older children to prepare fruit and vegetables themselves and stop cutting fruit and vegetables as snacks for them. Another possible explanation for the decline in consumption according to age is that young adolescents' eating habits are primarily under the influence of their parents, whereas the older adolescents are orientated towards their peers. Therefore, older schoolchildren will be more prone to peer pressure towards eating unhealthily. Finally, the teenagers' growth spurt may be another barrier to eating fruit and vegetables as they do not perceive fruit and vegetables as having enough filling power.

The fourth contribution of this review is some potential explanations as to why children in most western countries have a higher intake of fruit than vegetables [5,62]. Children's preference for fruit over vegetables appeared to be related to the sensory attributes of fruit and vegetables and to the way vegetables are prepared. Another barrier to vegetable intake may be children's perception of dinner as the only appropriate time and family/home as the only appropriate setting for eating vegetables, whereas fruit can be eaten everywhere at all times of the day. This is in agreement with the quantitative finding that frequency of family meals is associated positively with fruit and vegetable intake [11].
Finally, as a fifth contribution the review brings into focus the shortage of published, peer-reviewed qualitative studies from countries outside the US.

No previous systematic review of qualitative studies on children's fruit and vegetable intake is directly comparable to the present review. Jago et al. (2007) found qualitative support for an association between availability of fruit and vegetables and consumption of fruit and vegetables among children and adults in a systematic review of both qualitative and quantitative studies investigating the role of availability [63]. Based on findings from eight qualitative studies they suggested that availability was affected by SEP and location (rural/urban/reservation), but not ethnicity. Our review based on 31 studies is consistent with this [63]. Other systematic reviews among children have focused on determinants of healthy eating, are based on both peer-reviewed papers and grey literature (published and unpublished reports and theses) and have included both quantitative (non-experimental and intervention) and qualitative studies simultaneously $[18,64]$. Even though their findings are not specific to fruit and vegetable consumption, the facilitators and barriers to healthy eating overlap with many of the identified determinants of fruit and vegetable intake in our review. For example Shepherd et al. (2006) concluded that barriers to healthy eating among 11-16-year-olds were low access to and high cost of healthy food, time considerations and personal preferences for, and easy access to fast food. Facilitators for eating healthily were among other things concerns about appearances and parental support. Furthermore the young people associated fast food with friends and pleasure and valued the ability to choose what they wanted to eat [18]. The 4-10-year-olds in the review by Thomas et al. (2004) valued taste over health, said that "everything that is healthy tastes awful" (page 1011) and did not consider fruit and vegetables as the same kind of food [64]. Further the children perceived their parents as responsible for their personal health e.g. providing the children with fruit [64]. Other reviews in this area are mostly narrative reviews and have not applied systematic review methods to literature search, extraction of data, or quality assessment or been explicit about the methods they have applied [65-68].

\section{Study limitations and strengths}

Qualitative research is multidisciplinary with studies spread over multiple journals and databases. Several papers mention the difficulties in making exhaustive qualitative literature searches due to inconsistent indexing and use of search terms in databases as well as the lack of databases gathering qualitative health research [69-71]. We limited our search to qualitative research by using the search terms anthropology, anthropologic, 
anthropological, ethnography, ethnographic, ethnographical, qualitative, focus group, focus groups, and grounded theory. We did not use the search term 'interview' as questionnaire surveys are sometimes carried out as interviews. Our search strategy may have excluded qualitative research which has not been indexed correctly by our search terms. Two approaches were used to prevent this. Firstly, the literature search built upon the literature search for quantitative studies for part one of this review. For this literature search we used a less restricted combination of search terms with no requirements for study design ((fruit(s) or vegetables(s)) and ('children or adolescents')) in Medline and PsycINFO. This literature search yielded eight qualitative studies. All of these papers were also identified through our literature search for qualitative studies except a paper published in 1973 which was not indexed as a qualitative study [16]. Secondly, we checked our own records and screened the reference lists for relevant qualitative studies in all full text papers we retrieved for both reviews as well as reference lists of existing reviews. Only one of the 24 additional records we identified through these sources was included in the present review, namely the previous mentioned study from 1973 [16].

We only included peer-reviewed papers in English. These language and publication status restrictions may have posed a risk for publication bias. However, the findings presented in reviews which included grey literature were not in conflict with our conclusions [72,73]. Research published in languages other than English may contribute with important country-specific insight. A few non-English papers from Brazil, Canada, Chile, Germany, and Spain were excluded limiting the variation of settings in the review.

Qualitative findings and interpretations may be more difficult to report in other languages than the language used in the study as translation of quotations into English may result in loss of meaning due to national figures of speech. The fact that 27 out of 31 of the included papers in the review were conducted in English-speaking countries (the US, the UK, Ireland, New Zealand, and Australia) may support this assumption.

We only included findings about children's own views and perceptions of factors influencing their fruit and vegetable consumption and therefore the views of parents and school staff which were expressed in some of the studies included were left out of the summary of findings. Triangulation of sources may enhance the validity of qualitative studies by contributing with more perspectives and dimensions to the phenomenon studied e.g. there may be some constraints on children's intake that they do not perceive as barriers themselves, because other people (parents, school staff, parents of friends) are mainly responsible for increasing children's access to fruit and vegetables. For example parents in two of the studies included said that they expected their children to cut up fruit and vegetables for themselves or ask for help if necessary $[25,48]$ whereas one finding of this review was that children expected their parents to provide them with fruit snacks $[28,32,35,42]$. The inclusion of both views suggests a discrepancy between children's and parents' perception of their own role and responsibility in relation to increasing accessibility of fruit and vegetables at home.

In the two reviews we have aimed at mapping out all available peer-reviewed quantitative and qualitative evidence on potential determinants of fruit and vegetable consumption among children and adolescents irrespective of country. For the present review we performed a conceptual analysis to synthesise the findings. We analysed potential determinants/themes across studies conducted in different country settings (maximum variation sampling) and deliberately de-contextualised the findings from their original study setting. Any approach which de-contextualize information, e.g. by analysing themes across interviews or across studies instead of focusing on the uniqueness of individuals (case studies) or single studies runs the risk of losing sight of context [69]. To prevent this we could have chosen to make a similar review of qualitative studies within one country setting only (homogeneous sampling). However due to the limited number of qualitative studies identified for this review it would only be meaningful for a few countries such as the US where an abundant number of qualitative studies were conducted.

We evaluated the methodological quality of the papers in two ways. The quantitative approach was a count of quality criteria met. As we did not perceive all quality criteria as equally important for the overall quality, we also conducted an overall qualitative assessment similar to a peer-review process. Our experience was that both methods provided a useful insight into the scientific quality of the included papers. Further, that the quantitative approach constituted a useful basis for the overall qualitative assessment and that the validity of the qualitative approach was improved by obtaining agreement between at least two independent assessors. The qualitative approach is more open for interpretation and dependent on the reviewers' experience with qualitative research.

In the present study we made a comprehensive literature search in 14 electronic databases of biomedical and social science literature which makes it likely that we identified nearly all relevant peer-reviewed studies. Another strength of this study is the systematic and standardised procedures we used to review and evaluate the papers we included. These procedures enriched the review for example by 1 ) mapping out the variety of views on determinants expressed by children and adolescents across studies, and 
2) showing that low quality studies did not detect gender differences in outcome expectations as opposed to studies of high quality. A third strength is that we applied a broad inclusion to obtain the greatest understanding of fruit and vegetable consumption in adolescence [74]. We chose a priori to include all studies that fulfilled the inclusion criteria irrespective of methodological quality. Further, we aimed to present a variety of views and not only those that were mentioned by most children or most focus groups. This approach is in agreement with Fade (2003) who states that "qualitative analysis is not simply a case of counting up the number of times a view is expressed and presenting the most frequently expressed view" (page 144) [22].

\section{Conclusions and recommendations for research}

This review reveals a series of potential determinants of fruit and vegetable intake among children of which our current knowledge is limited (table 3). The prevalence, relative importance and explanatory power of these factors must be assessed in future large scale quantitative studies as well as their interplay with other important determinants such as taste preferences and home availability. The qualitative studies suggest for example, that even when children have a high taste preference for fruit and vegetables they will not eat them if it is not convenient. Even though taste preferences and home availability are necessary prerequisites for children's fruit and vegetable consumption, they may not be sufficient factors. Interactions between home availability, taste preferences, convenience (home accessibility, parental facilitation) and fruit and vegetable consumption should be studied in multivariate analyses.

The concept of availability is often treated as onedimensional representing whether fruit and vegetables are present at home or in school or not. This review however, suggests that availability is a multidimensional construct and the relative importance of different dimensions of home, school and local area availability such as presence, variety, visibility, quality, texture, cost, convenience, time, access to competitive unhealthy food and methods of preparation should be examined in epidemiological surveys.

Understanding the mechanisms behind the clear sociodemographic differences in fruit and vegetable intake is an important area for future research. The suggested mechanisms from this review should be tested in epidemiological surveys. Such studies may guide the development of subgroup-specific intervention strategies. Future studies should for example clarify the relative importance of different aspects of outcome expectancies of eating fruit and vegetables for consumption among boys and girls and in different age-groups such as positive versus negative expectations, short and long term expectations, and health related versus social outcome expectations (such as expecting parental reward if eating fruit and vegetables).

In the sensitivity analysis we only examined the influence of methodological quality of the included studies on the derived themes. As part of sensitivity analysis, future reviews should also analyse the influence of other study characteristics such as country setting or sociodemographic characteristics of study population.

The review highlights peer influences as important for fruit and vegetable consumption especially among older children and girls. However, peer pressure and peer influences are hard to capture. Qualitative studies and validation studies should aim at conceptualising and operationalising measures of peer support and peer pressure towards eating unhealthily and healthily in order to strengthen future surveys among school-aged children. Multivariate models should examine SEP as a potential effect-modifier for the interplay between exposure to a large variety of fruit and vegetables at home, taste preferences and fruit and vegetable consumption. Furthermore this review confirms that determinants for fruit and vegetable intake should be analysed separately. Children's preference for fruit over vegetables has mainly been explained by taste. This review suggests that other potential barriers for vegetable intake are methods of preparation, texture, appearance and children's ideas of when, where and with whom it is appropriate to eat vegetables. This should be tested quantitatively. Finally, most of the included studies in this review were from the US. Future qualitative studies should explore if the results of this review are transferable to other country settings or if other factors are more important in shaping children's fruit and vegetable consumption there.

\section{Conclusions and recommendations for practice}

This review of qualitative studies emphasises the importance of convenience and the trade off between time and being healthy - issues that could be transferred into product development. Children prefer snacks and chocolate bars, because they are instantly available, can be eaten straight away, do not require any time-consuming preparation, can be carried in your school bag without getting squashed and soggy and because the taste and quality is guaranteed so they do not risk wasting their money on bad quality. These characteristics of snacks can inspire interventions at the family, school and societal level. At the family level, parents should prepare (or assist children in preparing) fruit and vegetables as readily available snacks in between meals (peeled and cut into bite-sized pieces). At the school level, schools could encourage children to eat fruit and vegetables by having a fruit break, where children are allocated time to prepare and eat fruit and vegetables, by offering fresh appealing fruit and vegetables of high quality and variety in the school canteen 
and refrigerators where the children can store their fruit and vegetables brought from home. At the industrial level, producers should be encouraged to sell fresh (not canned) appealing fruit and vegetable snacks, peeled, cut and ready to eat as a worthy alternative to unhealthy snacks. Super markets should give children visual cues to eat fruit and vegetables by placing the fruit and vegetables near the counter and by advertising them on posters and signs. Furthermore, the availability of less healthy rival food choices should be limited and hidden away from children.

This review has demonstrated the potential of qualitative studies to illustrate the views of children on barriers and facilitators to eating fruit and vegetables. The reviews of qualitative and quantitative studies are not mutually exclusive but complement each other to give a more comprehensive understanding of determinants for children's fruit and vegetable intake. The low number of qualitative studies identified is striking. It is common to do qualitative pilot studies before conducting larger quantitative surveys, but those results are seldom published in scientific journals. However, this review shows that the information gained from qualitative studies is very valuable and researchers should be encouraged to publish results from these studies.

\section{Additional material}

\section{Additional file 1: Quality assessment tool for systematic review of} qualitative studies. Description of quality indicators used in the quality assessment scheme (table 2). Appendix to Krølner et al. Determinants of fruit and vegetable consumption among children and adolescents: a review of the literature. Part II: qualitative studies. Int J Behav Nutr Phys Act 2011 8:112 doi:10.1186/1479-5868-8-112 (See also table 2 in the paper)

\section{Acknowledgements}

The authors thank Professor Bjørn Holstein for providing helpful comments to the manuscript and for acting as peer debriefer (discuss inconsistencies in analysis and interpretation of data between reviewers). Julie Aarestrup, graduate student in human nutrition, assisted in obtaining full-text articles of potentially relevant citations from literature searches. The authors wish to thank the anonymous reviewers for their constructive comments. Financial support

The Danish Medical Research Council, The Faculty of Health Sciences at the University of Copenhagen, The Tryg Foundation, the Nordea Foundation The idea for this study originated in the Pro Children project. The Pro Children study was carried out with financial support from the Commission of the European Communities, specific RTD programme "Quality of Life and Management of Living Resources", QLK1-2001-00547 "Promoting and sustaining health through increased fruit and vegetable consumption among European schoolchildren" (Pro Children). The study does not necessarily reflect the Commission's views and in no way anticipates the Commission's future policy in this area.

\section{Author details}

${ }^{1}$ National Institute of Public Health, University of Southern Denmark, Copenhagen, Denmark. ${ }^{2}$ Department of Epidemiology and Biostatistics and the EMGO Institute for Health and Care research, VU University Medical Center, Amsterdam, The Netherlands. ${ }^{3}$ Department of Nutrition, Faculty of
Medicine, University of Oslo, Norway. ${ }^{4}$ GGD Gelre-IJssel (Community Health Service), Apeldoorn, The Netherlands.

\section{Authors' contributions}

RK conceived the study, designed and directed the analysis, carried out the literature search data extraction and analysis of the papers included, and drafted the manuscript. MR conceived the study, participated in the design of the analysis and in the selection of papers for inclusion and analysis of the papers included, contributed to the interpretation of data and revised the manuscript critically. JB conceived the study, contributed to the interpretation of data and revised the manuscript critically. KIK conceived the study, contributed to the interpretation of data and revised the manuscript critically. MW contributed to the interpretation of data and revised the manuscript critically. PD conceived the study, participated in the design of the analysis and in the selection of papers for inclusion and analysis of the papers included, contributed to the interpretation of data and revised the manuscript critically. All authors read and approved the final manuscript.

\section{Competing interests}

The authors declare that they have no competing interests.

Received: 6 June 2011 Accepted: 14 October 2011

Published: 14 October 2011

\section{References}

1. He FJ, Nowson CA, MacGregor GA: Fruit and vegetable consumption and stroke: meta-analysis of cohort studies. Lancet 2006, 367:320-326.

2. He FJ, Nowson CA, Lucas M, MacGregor GA: Increased consumption of fruit and vegetables is related to a reduced risk of coronary heart disease: meta-analysis of cohort studies. J Hum Hypertens 2007, 21:717-728.

3. Van Duyn MA, Pivonka E: Overview of the health benefits of fruit and vegetable consumption for the dietetics professional: selected literature. J Am Diet Assoc 2000, 100:1511-1521.

4. Guenther PM, Dodd KW, Reedy J, Krebs-Smith SM: Most Americans eat much less than recommended amounts of fruits and vegetables. J Am Diet Assoc 2006, 106:1371-1379.

5. Yngve A, Wolf A, Poortvliet E, Elmadfa I, Brug J, Ehrenblad B, Franchini B, Haraldsdóttir J, Krølner R, Maes L, Pérez-Rodrigo C, Sjöström M, Thórsdóttir I, Klepp Kl: Fruit and vegetable intake in a sample of 11-year-old children in 9 European countries: The Pro Children Cross-sectional Survey. Ann Nutr Metab 2005, 49:236-245.

6. Vereecken CA, De Henauw S, Maes L: Adolescents' food habits: results of the Health Behaviour in School-aged Children survey. Br J Nutr 2005, 94:423-431.

7. Kelder SH, Perry CL, Klepp KI, Lytle LL: Longitudinal tracking of adolescent smoking, physical activity, and food choice behaviors. Am J Public Health 1994, 84:1121-1126.

8. Lien N, Lytle LA, Klepp Kl: Stability in consumption of fruit, vegetables, and sugary foods in a cohort from age 14 to age 21. Prev Med 2001, 33:217-226.

9. Te Velde SJ, Twisk JW, Brug J: Tracking of fruit and vegetable consumption from adolescence into adulthood and its longitudinal association with overweight. Br J Nutr 2007, 98:431-438.

10. Dixon-Woods M, Fitzpatrick R, Roberts $K$ : Including qualitative research in systematic reviews: opportunities and problems. J Eval Clin Pract 2001, 7:125-133.

11. Rasmussen M, Krølner R, Klepp Kl, Lytle L, Brug J, Bere E, Due P: Determinants of fruit and vegetable consumption among children and adolescents: a review of the literature. Part I: quantitative studies. Int $J$ Behav Nutr Phys Act 2006, 3:22.

12. Kuzel AJ: Sampling in qualitative inquiry. In Doing qualitative research. Edited by: Crabtree BF, Miller WL. Thousand Oaks: Sage Publications, Inc.; 1999:33-45.

13. Pearson N, Biddle SJ, Gorely T: Family correlates of fruit and vegetable consumption in children and adolescents: a systematic review. Public Health Nutr 2009, 12:267-283.

14. Kamphuis CB, Giskes K, de Bruijn GJ, Wendel-Vos W, Brug J, van Lenthe FJ: Environmental determinants of fruit and vegetable consumption among adults: a systematic review. Br J Nutr 2006, 96:620-635. 
15. van der Horst K, Oenema A, Ferreira I, Wendel-Vos W, Giskes K, van Lenthe F, Brug J: A systematic review of environmental correlates of obesity-related dietary behaviors in youth. Health Educ Res 2007, 22:203-226.

16. Walker MA, Hill MM, Millman FD: Fruit and vegetable acceptance by students. Factors in acceptance and performance. J Am Diet Assoc 1973, 62:268-272.

17. Harden A, Garcia J, Oliver S, Rees R, Shepherd J, Brunton G, Oakley A: Applying systematic review methods to studies of people's views: an example from public health research. J Epidemiol Community Health 2004, 58:794-800.

18. Shepherd J, Harden A, Rees R, Brunton G, Garcia J, Oliver S, Oakley A: Young people and healthy eating: a systematic review of research on barriers and facilitators. Health Educ Res 2006, 21:239-257.

19. Pound P, Britten N, Morgan M, Yardley L, Pope C, Daker-White G, Campbell R: Resisting medicines: a synthesis of qualitative studies of medicine taking. Soc Sci Med 2005, 61:133-155.

20. Mills E, Jadad AR, Ross C, Wilson K: Systematic review of qualitative studies exploring parental beliefs and attitudes toward childhood vaccination identifies common barriers to vaccination. J Clin Epidemiol 2005, 58:1081-1088.

21. Malterud K: Qualitative research: standards, challenges, and guidelines. Lancet 2001, 358:483-488.

22. Fade SA: Communicating and judging the quality of qualitative research: the need for a new language. J Hum Nutr Diet 2003, 16:139-149.

23. Kvale S, Brinkmann S: The social construction of validity. In Interviews Learning the craft of qualitative research interviewing. Edited by: Kvale S, Brinkmann S. Thousand Oaks: Sage publications, inc.; 2008:241-266.

24. Strauss A, Corbin J: Basics of qualitative research. Techniques and procedures for developing grounded theory. 2 edition. Thousand Oaks: Sage Publications; 1998.

25. Cullen KW, Baranowski T, Baranowski J, Warnecke C, de Moor C, Nwachokor A, Hajek RA, Jones LA: "5 A Day" achievement badge for urban boy scouts: formative evaluation results. J Cancer Educ 1998, 13:162-168.

26. Cullen KW, Hartstein J, Reynolds KD, Vu M, Resnicow K, Greene N, White MA: Improving the school food environment: results from a pilot study in middle schools. J Am Diet Assoc 2007, 107:484-489.

27. Nicklas TA, Johnson CC, Farris R, Rice R, Lyon L, Shi R: Development of a school-based nutrition intervention for high school students: Gimme 5. Am J Health Promot 1997, 11:315-322.

28. Monge-Rojas R, Garita C, Sanchez M, Munoz L: Barriers to and motivators for healthful eating as perceived by rural and urban Costa Rican adolescents. J Nutr Educ Behav 2005, 37:33-40.

29. Baranowski T, Domel S, Gould R, Baranowski J, Leonard S, Treiber F, Mullis R: Increasing fruit and vegetable consumption among 4th and 5th grade students - Results from focus groups using reciprocal determinism. J Nutr Educ 1993, 25:114-120.

30. Campbell E: Dietary choices of urban minority high school students. Pediatr Nurs 2009, 35:171-180

31. Gellar LA, Schrader K, Nansel TR: Healthy eating practices: perceptions, facilitators, and barriers among youth with diabetes. Diabetes Educ 2007, 33:671-679.

32. Hill L, Casswell S, Maskill C, Jones S, Wyllie A: Fruit and vegetables as adolescent food choices in New Zealand. Health Promot Int 1998, 13:55-65.

33. Keim KS, Swanson MA, Cann SE: Caucasian and Mexican American lowincome children's thoughts about vegetables and fruits. Ecol Food Nutr 2001, 40:525-544.

34. Kirby SD, Baranowski T, Reynolds KD, Taylor G, Binkley D: Children's fruit and vegetable intake - Socioeconomic, adult-child, regional, and urbanrural influences. J Nutr Educ 1995, 27:261-271.

35. Kubik MY, Lytle L, Fulkerson JA: Fruits, vegetables, and football: findings from focus groups with alternative high school students regarding eating and physical activity. J Adolesc Health 2005, 36:494-500.

36. Lautenschlager $L$, Smith $C$ : Beliefs, knowledge, and values held by innercity youth about gardening, nutrition, and cooking. Agric Human Values 2007, 24:245-258.

37. Molaison EF, Connell CL, Stuff JE, Yadrick MK, Bogle M: Influences on fruit and vegetable consumption by low-income black American adolescents. J Nutr Educ Behav 2005, 37:246-251.
38. Wind M, Bobelijn K, De Bourdeaudhuij I, Klepp Kl, Brug J: A qualitative exploration of determinants of fruit and vegetable intake among 10 and 11-year-old schoolchildren in the low countries. Ann Nutr Metab 2005, 49:228-235.

39. Zeinstra GG, Koelen MA, Kok FJ, de Graaf C: Cognitive development and children's perceptions of fruit and vegetables; a qualitative study. Int J Behav Nutr Phys Act 2007, 4:30.

40. Evans AE, Wilson DK, Buck J, Torbett H, Williams J: Outcome expectations, barriers, and strategies for healthful eating - A perspective from adolescents from low-income families. Fam Community Health 2006, 29:17-27.

41. Fitzgerald E, Bunde-Birouste A, Webster E: Through the eyes of children: engaging primary school-aged children in creating supportive school environments for physical activity and nutrition. Health Promot J Austr 2009, 20:127-132.

42. Kim LP, Harrison GG, Kagawa-Singer M: Perceptions of diet and physical activity among California Hmong adults and youths. Prev Chronic Dis 2007, 4:A93.

43. Libman K: Growing youth growing food: How vegetable gardening influences young people's food consciousness and eating habits. Appl Environ Educ Comm Int J 2007, 6:87-95.

44. McKinley MC, Lowis C, Robson PJ, Wallace JM, Morrissey M, Moran A, Livingstone MB: It's good to talk: children's views on food and nutrition. Eur J Clin Nutr 2005, 59:542-551.

45. Neumark-Sztainer D, Story M, Perry C, Casey MA: Factors influencing food choices of adolescents: findings from focus-group discussions with adolescents. J Am Diet Assoc 1999, 99:929-937.

46. Ross S: "Do I really have to eat that?": a qualitative study of schoolchildren's food choices and preferences. Health Educ J 1995, 54:312-321.

47. Stevenson C, Doherty G, Barnett J, Muldoon OT, Trew K: Adolescents' views of food and eating: identifying barriers to healthy eating. J Adolesc 2007, 30:417-434.

48. Cullen KW, Baranowski T, Rittenberry L, Olvera N: Social-environmental influences on children's diets: results from focus groups with African-, Euro- and Mexican-American children and their parents. Health Educ Res 2000, 15:581-590.

49. Booth ML, Wilkenfeld RL, Pagnini DL, Booth SL, King LA: Perceptions of adolescents on overweight and obesity: the weight of opinion study. J Paediatr Child Health 2008, 44:248-252.

50. Khunti K, Stone MA, Bankart J, Sinfield P, Pancholi A, Walker S, Talbot D, Farooqi A, Davies MJ: Primary prevention of type-2 diabetes and heart disease: action research in secondary schools serving an ethnically diverse UK population. J Public Health (Oxf) 2008, 30:30-37.

51. Husby I, Heitmann BL, O'Doherty JK: Meals and snacks from the child's perspective: the contribution of qualitative methods to the development of dietary interventions. Public Health Nutr 2009, 12:739-747.

52. O'dea JA: Why do kids eat healthful food? Perceived benefits of and barriers to healthful eating and physical activity among children and adolescents. J Am Diet Assoc 2003, 103:497-501.

53. Goh YY, Bogart LM, Sipple-Asher BK, Uyeda K, Hawes-Dawson J, OlaritaDhungana J, Ryan GW, Schuster MA: Using community-based participatory research to identify potential interventions to overcome barriers to adolescents' healthy eating and physical activity. J Behav Med 2009, 32:491-502.

54. Kubik MY, Lytle LA, Hannan PJ, Perry CL, Story M: The association of the school food environment with dietary behaviors of young adolescents. Am J Public Health 2003, 93:1168-1173.

55. Hearn MD, Baranowski T, Baranowski J, Doyle C, Smith M, Lin LS, Resnicow K: Environmental influences on dietary behavior among children: availability and accessibility of fruits and vegetables enable consumption. J Health Educ 1998, 29:26-32.

56. De Bourdeaudhuij I, Klepp Kl, Due P, Pérez Rodrigo C, de Almeida MDV, Wind M, Krølner R, Sandvik C, Brug J: Reliability and validity of a questionnaire to measure personal, social and environmental correlates of fruit and vegetable intake in 10-11-year-old children in five European countries. Public Health Nutr 2005, 8:189-200.

57. Bauer KW, Yang YW, Austin SB: "How can we stay healthy when you're throwing all of this in front of us?" Findings from focus groups and interviews in middle schools on environmental influences on nutrition and physical activity. Health Educ Behav 2004, 31:34-46. 
58. McClain AD, Chappuis C, Nguyen-Rodriguez ST, Yaroch AL, Spruijt-Metz D: Psychosocial correlates of eating behavior in children and adolescents: a review. Int I Behav Nutr Phys Act 2009, 6:54.

59. Blanchette L, Brug J: Determinants of fruit and vegetable consumption among 6-12-year-old children and effective interventions to increase consumption. J Hum Nutr Diet 2005, 18:431-443.

60. Resnicow K, Davis-Hearn M, Smith M, Baranowski T, Lin LS, Baranowski J, Doyle C, Wang DT: Social-cognitive predictors of fruit and vegetable intake in children. Health Psychol 1997, 16:272-276.

61. de Sa J, Lock K: Will European agricultural policy for school fruit and vegetables improve public health? A review of school fruit and vegetable programmes. Eur J Public Health 2008, 18:558-568.

62. Currie C, Roberts C, Morgan A, Smith R, Settertobulte W, Samdal O, Rasmussen VB, (eds): Young People's Health in Context: international report from the HBSC 2001/02 survey. WHO Policy Series: Health policy for children and adolescents Issue 4 Copenhagen: WHO Regional Office for Europe; 2004.

63. Jago R, Baranowski T, Baranowski JC: Fruit and vegetable availability: a micro environmental mediating variable? Public Health Nutr 2007, 10:681-689.

64. Thomas J, Harden A, Oakley A, Oliver S, Sutcliffe K, Rees R, Brunton G, Kavanagh J: Integrating qualitative research with trials in systematic reviews. BMJ 2004, 328:1010-1012.

65. Jenkins S, Horner SD: Barriers that influence eating behaviors in adolescents. J Pediatr Nurs 2005, 20:258-267.

66. Taylor JP, Evers S, McKenna M: Determinants of healthy eating in children and youth. Can J Public Health 2005, 96(Suppl 3):S20-S29.

67. Shepherd R, Dennison CM: Influences on adolescent food choice. Proc Nutr Soc 1996, 55:345-357.

68. Patrick $\mathrm{H}$, Nicklas TA: A review of family and social determinants of children's eating patterns and diet quality. J Am Coll Nutr 2005, 24:83-92.

69. Dixon-Woods M, Bonas S, Booth A, Jones DR, Miller T, Sutton AJ, Shaw RL, Smith $J A$, Young B: How can systematic reviews incorporate qualitative research? A critical perspective. Qual Res 2006, 6:27-44.

70. McKibbon KA, Wilczynski NL, Haynes RB: Developing optimal search strategies for retrieving qualitative studies in PsycINFO. Eval Health Prof 2006, 29:440-454.

71. Barroso J, Gollop CJ, Sandelowski M, Meynell J, Pearce PF, Collins LJ: The challenges of searching for and retrieving qualitative studies. West J Nurs Res 2003, 25:153-178.

72. Shepherd J, Harden A, Rees R, Brunton G, Garcia J, Oliver S, Oakley A: Young people and healthy eating: a systematic review of research on barriers and facilitators. London: EPPI-Centre, Social Science Research Unit, Institute of Education, University of London; 2001.

73. Thomas J, Sutcliffe K, Harden A, Oakley A, Oliver S, Rees R, Brunton G, Kavanagh J: Children and healthy eating: a systematic review of barriers and facilitators. London: EPPI-Centre, Social Science Research Unit, Institute of Education, University of London; 2003.

74. Jensen LA, Allen MN: Meta-synthesis of qualitative findings. Qual Health Res 1996, 6:553-560.

doi:10.1186/1479-5868-8-112

Cite this article as: Krølner et al:: Determinants of fruit and vegetable consumption among children and adolescents: a review of the literature. Part II: qualitative studies. International Journal of Behavioral Nutrition and Physical Activity 2011 8:112.

\section{Submit your next manuscript to BioMed Central and take full advantage of:}

- Convenient online submission

- Thorough peer review

- No space constraints or color figure charges

- Immediate publication on acceptance

- Inclusion in PubMed, CAS, Scopus and Google Scholar

- Research which is freely available for redistribution

Submit your manuscript at www.biomedcentral.com/submit
Ciomed Central 